\title{
PERMEATION OF POLYELECTROLYTES AND OTHER SOLUTES INTO THE PORE SPACES OF WATER-SWOLLEN CELLULOSE: A REVIEW
}

\author{
Ning Wu, Martin A. Hubbe, * Orlando J. Rojas, and Sunkyu Park \\ The rate and extent of transport of macromolecules and other solutes \\ into cellulosic materials and fibers have important applications in such \\ fields as papermaking, textiles, medicine, and chromatography. This \\ review considers how diffusion and flow affect permeation into wood, \\ paper, and other lignocellulosic materials. Because pore sizes within \\ such materials can range from nanometers to millimeters, a broad \\ perspective will be used, also considering some publications related to \\ other porous materials. Factors that limit the rate or extent of polymer or \\ other solute transport into pores can involve thermodynamics (affecting \\ the driving motivation for permeation), kinetics (if there is insufficient time \\ for the system to come to equilibrium), and physical barriers. Molecular \\ flow is also affected by the attributes of the solute, such as molecular \\ mass and charge, as well as those of the substrate, such as the pore \\ size, interconnectedness, restricted areas, and surface characteristics. \\ Published articles have helped to clarify which of these factors may have \\ a controlling influence on molecular transport in different situations.
}

Keywords: Diffusion; Flow; Permeation; Porous materials; Cellulose; Polyelectrolytes; Solutes; Enablers

Contact information: Department of Forest Biomaterials, North Carolina State University, Campus Box 8005; Raleigh, NC 29695-8005. *Corresponding author: hubbe@ncsu.edu

\section{INTRODUCTION}

Permeation of macromolecules and other dissolved or suspended matter from aqueous solution into cellulosic material is important in the manufacture of paper, in the sizing of textile fibers, in filtration, in various types of absorption processes, and in the manufacture of cellulosic composites or nanocomposites. In each of these domains it is important to understand factors that affect the degree to which molecules, ions, surfactants, polyelectrolytes, or colloids penetrate into the interior pores of lignocellulosic matter. Polyelectrolytes are of particular interest. In papermaking, often it is considered advantageous for polyelectrolytes to remain at or near to the outer surface of cellulosic fibers, thereby maximizing their contributions to various processes, such as fine-particle retention, dewatering, or the development of inter-fiber bonding. In the textile industry surface-finishing with polymers and other additives is important for fiber processing and use. In bioethanol production the diffusion of enzymes determines the efficiency with which cellulose hydrolysis can take place. In the wood industry it is important to understand the permeation of preservatives, components of surface finishes, and even of rot-related enzymes. 
In many applications there is an advantage if one can achieve faster penetration of polymeric materials into the pores of cellulosic materials. This is certainly true in the case of glue penetration for wood-based construction (Kamke and Lee 2007). Material transport by convection and diffusion, as well as by fluid penetration are essential steps for chemicals to interact with wood chips during production cellulosic pulps (Gustafson et al. 1997). The interior part of the wood chips is not easily reached by chemicals. Thus, short contact times of wood chips with reactive liquor may result in non-uniform cooking of cellulose pulp. Polymer permeation phenomena also are important in fields such as enhanced oil recovery; polymers may be used to influence the channeling of flow through oil-containing strata (Babadagli 2007).

Publications considered in this review article provide evidence of factors and mechanisms that affect the rate or extent to which solute transport occurs into the fine pores of cellulosic materials. The review takes advantage of the fact that relevant work also has been carried out with such materials as silica gel, mineral beds, and plastic materials. Emphasis will be placed on charged polymers, especially in cases where opposite charges contribute to a driving force favoring permeation. For completeness, such situations will be contrasted with cases where adsorption may be favored by changes in entropy accompanying permeation and adsorption. Both bulk transport by convection and molecular diffusion will be considered. Aspects of the fine-scale porosity, pore sizes, and interconnectedness of pores within cellulosic materials (tortuosity) also will be considered.

At the outset it is important to acknowledge several prior review articles, many of which go deeper than the present article in different areas that may be of interest to particular readers (Stamm 1953; Stone and Green 1958; Siau 1984; Robertson and Lönnberg 1991a,b; Lönnberg et al. 1992; Muralidhar and Ramkrishna 1993; Sahimi 1993; Starov 2004; Dechadilok and Deen 2006; Delgado 2006; Babadagli 2007; Johnson et al. 2007; Kamke and Lee 2007; Chavez-Rojo et al. 2008). Also it is worth noting several articles dealing with nonuniformities in transport rates through packed columns, which can result in "tails" in eluted solutes (Newman and Walker 1992; Kosakowski 2004; Obi and Blunt 2004; Delgado 2006).

\section{Organization of this Article}

This article is organized into three main sections, the first of which deals with factors that can affect permeation. Emphasis is placed on publications reporting practical observations. Factors affecting permeation, relating to the nature of the pores, the fluids, and interactions at interfaces, tend to apply for a broad range of applications; therefore citations will be considered from diverse fields.

The next main section considers mechanisms of permeation, with emphasis on flow and diffusion. Flow can be defined as movement of a fluid phase relative to its surroundings. Diffusion can be defined as a mechanism by which transport is driven by a gradient in concentration. In many practical situations transport of solutes will result from a combination of diffusion and fluid motion. Flow is important in cases where liquid moves either into or through a porous material; in such cases the moving fluid may or may not be able to carry along various dissolved or entrained substances. As will be 
discussed, barriers related to entropy, charge, or physical inaccessibility may tend to exclude the polymeric material in some instances.

Realizing that certain readers may have an interest in promoting access of polyelectrolytes, enzymes, or other solutes into the interior spaces of cellulosic materials, a third section of this article will be devoted to "enablers" of permeation. An enabler is defined here as anything that promotes either more rapid or more complete penetration, either by flow or by diffusion through aqueous solution.

\section{OVERVIEW OF FACTORS AFFECTING DIFFUSION AND FLOW IN PORES}

Various factors can affect the progress of dissolved or very finely suspended substances into porous media. These factors generally can be grouped into the pore attributes, the nature of the fluid (carrier), and the interactions between the fluid medium and the solids, including any dispersed substances (soluble, colloidal, or particulate) (Lönnberg et al. 1992; Lebon et al. 2006). The complexity of diffusion and flow in pores involves many branches of science, of which thermodynamics deserves special attention. Variables of interest for diffusion and flow in pores include the set of classical variables, e.g. mass, momentum, and heat, as well as the corresponding fluxes of these quantities.

\section{Pore Attributes: Case of Cellulosic Materials}

Cellulose is a porous material, especially when it is wet with water. Many applications of cellulose involve its interaction with fluids that flow or diffuse into its porous structure. Knowing the physical-chemical structures and attributes of these pores helps to understand associated interactions of cellulose' surface with fluids and provides guidance for related applications. For instance, Kamke and Lee (2007) have considered many such factors in their review of adhesive penetration into wood.

\section{Pore size and size distribution (morphology)}

Permeability of cellulosic materials can be expected to increase with increasing pore size or with the removal of obstructions from pores. For instance, alkaline conditions tend to promote more rapid penetration into cellulosic materials (Scallan 1983); this has been attributed to increased swelling, an effect that can increase the sizes of pores.

There are at least two classes of pores within different types of cellulosic materials. Relatively large pores, often in the range of 0.1 to $100 \mu \mathrm{m}$, may exist either between the fibers (as in the case of paper products) or inside the fibers (i.e. the uncollapsed lumens of fibers in wood). Relatively small pores will be present within the cell walls of the fibers. The sizes of the latter pores have been the subject of considerable interest and discussion. Elegant work by means of the solute exclusion principle was used as evidence for pores in the cell walls of never-dried sulfite fibers having widths generally in the range of 2 to $20 \mathrm{~nm}$ (Stone and Scallan 1968). However, these estimates were later disputed as being too low, since the analysis was said not to take into account various entropic factors (Alince and van de Ven 1997). Based on adsorption of polyelectrolytes, the latter authors concluded that the pores in never-dried chemical pulps

Wu et al. (2009). "Polyelectrolyte permeation in cellulose," BioResources 4(3), 1222-1262. 1224 
should mostly be larger, possibly 40 to $100 \mathrm{~nm}$. Analyses of penetration into cellulosic materials also need to take into account the effects that the diffusing solution may have on the swelling, and thus the pore sizes within the material.

Senden et al. (2000) studied the effects of pore morphology on penetration rates for ink vehicles. In printing operations the setting of inks often involves the passing of a vehicle though a surface of a paper. In such cases a higher amount of micro-pores near the surface of paper may allow more ink pigments to be held near the paper surface, and this would be expected to result in a higher density of the printed image. It was noted that different micro-pore systems imbibe liquids at different rates, depending on their geometries. For instance, converging pore features can promote more rapid permeation, whereas sharply diverging features, as one passes toward the interior of a porous material, can act as blocks to capillary penetration.

\section{Pore Volume}

The fractional porosity can be defined as the ratio of the voids volume to the total volume of that material. In other words, porosity represents the storage capacity. However, a high porosity doesn't necessarily mean that the material has a high permeability, since the void spaces may be either interconnected or isolated. To give an example from geology, shales tend to have high porosity but very low permeability due to the poorly interconnected voids. By contrast, sandstones generally can have both high porosity and high permeability.

In the case of cellulosic materials, the pores may be between fibers, within the fiber walls, or consisting of the fiber lumens and their connections at pit openings (Stamm 1953). During wood's growth, pores can serve as a medium for transport of water and other nutrients. Pits can become narrowed due to drying, and they also can become choked to various degrees by wood resins. They also enable chemical diffusion and penetration during pulping. In the case of hardwood pulps flow through vessels is expected to be more important than flow through fiber lumens and pits (Stone and Green 1958).

Flow also can occur through the cell walls of cellulosic materials, especially when the material is water-saturated. The mesopores within cellulosic material tend to become somewhat enlarged during pulping operations due to the combined influences of swelling of the material and removal of lignin-rich domains, leaving behind the spaces once occupied by these domains (Berthold and Salmén 1997). The shapes and connections within the pore structure can be expected to change also as a result of mechanical refining of cellulosic pulps. When the pulp has been dried, some of pores will be irreversibly closed (Stone and Scallan 1966).

\section{Dimensions of the substrate}

Much can be learned about the porosity of wood by considering the dimensions of a typical wood chip used in pulping operations (Gustafson et al. 1997; Maattanen and Tikka 2008) or in a dilute acid pretreatment step in the production of cellulosic biofuels (Kim and Lee 2002). A wood chip for use in pulping often has a thickness in the range of 2-10 mm, whereas the length and breadth will be much larger (Schultz et al. 1999). The thinness of chips is intended so that pulping reagents can more-or-less completely 
penetrate the wood material before the onset of rapid chemical reaction. The thickness of chips has to be small enough so that diffusion in not the rate-limiting factor. Over-thick chips can be rechipped in order to overcome such problems (Maattanen and Tikka 2008).

\section{Tortuosity}

Tortuosity can be loosely defined as the ratio between the average distance that fluid has to travel to get through a material, compared to an imaginary straight-line distance. One way to quantify tortuosity of a non-conductive porous solid is by measuring the net conductivity when it is saturated with a salt solution of known bulk conductivity (Barrande et al. 2007). There also have been attempts at numerical simulation of tortuous pathways through a compact bed of particles (Ito et al. 2003). High tortuosity generally means that there are many twists and turns within the pore spaces.

In the case of pine and aspen wood, Jacobson and Banerjee (2006) estimated a tortuosity of 1.6, relative to the diffusion of the same aqueous solution in the absence of wood. A somewhat higher value for aspen, relative to pine was attributed to a finer fiber structure. Leyvaramos and Geankoplis (1994) obtained a tortuosity value of 3.5 for activated carbon, a material that can retain various structural features related to its origin as wood.

The effective tortuosity of a material can be strongly dependent on what one chooses as a probe to measure tortuosity. For instance, in one case Olson et al. (2005) observed that the tortuosity of a packed bed was about 40 times greater for bacteria, in comparison with the suspending medium. Though these observations might have other contributing explanations, it is reasonable to expect that relatively large particles or cells in a fluid medium might not be able to take advantage of the most direct routes through a porous material, depending on the dimensions of the most constricted passageways. Törnqvist et al. $(2001,2004)$ measured the progress of $\mathrm{HS}^{-}$ions through individual wood chips, when pulping liquor was placed on one side of the chip and deionized water was placed on the other. Though the movement of the front of ions (the drift speed) was similar to what was predicted by ion diffusions rates, there also was evidence that the nature of the pulping chemicals changed to degree of swelling of the pores in the chip.

\section{Pore orientation}

Liquid diffusion through porous material may also be related to the orientation of pores. This is particularly true for wood materials. Thus, Ra et al. (2001) studied the unsteady-state diffusion of boron through southern pine (Pinus spp.) at various moisture contents, temperatures, and treatment conditions. The fastest rates of diffusion were observed in the longitudinal direction, followed by the radial and the tangential directions. They reported longitudinal diffusion coefficients 10 to 20 times larger than the radial diffusion coefficients, and radial diffusion coefficients two to four times larger than the tangential diffusion coefficients. The diffusion rate increased with time in all directions. The longitudinal diffusion rate increased rapidly with moisture content (MC), while a slow increase in the radial diffusion rate was observed at MCs above $90 \%$. Temperature was found to have a more pronounced effect than MC. In later work by the same authors Ra et al. (2002) investigated constant radial diffusion coefficients of boron

Wu et al. (2009). "Polyelectrolyte permeation in cellulose," BioResources 4(3), 1222-1262. 1226 
through southern pine (Pinus spp.). The concentration profile (the total amount of boron diffusing through wood and the amount of boron remaining on the wood surface after different diffusion periods) of the treated samples was measured using a slicing technique.

Directional dependencies have been observed also in the case of pressure-induced flows through wood material. Stamm (1953) cited studies showing that flow in the direction of fibers can be 50 to 200 times greater than flow perpendicular to the fiber orientation under matched conditions. Sernek et al. (1999) studied the penetration of a liquid urea-formaldehyde adhesive (UF) into beech (Fagus sylvatica L.) wood. They found that penetration in the tangential direction was greater than in the radial direction. They also concluded that external pressure can expedite penetration.

Another factor that can affect permeability of a porous material in different directions is the presence of channels, cracks, or fractures (Wallstrom and Lindberg 1999; Zvikelksy and Weisbrod 2006; Maatinen and Tikka 2008). It has been shown that a disproportionate amount of fluid may pass through such favorable routes, especially when one is concerned with colloidal matter (Kjaergaard et al. 2004a).

\section{Interconnectedness}

Pores can exist individually, or they may be interconnected to various extents. Interconnectedness makes it more difficult to interpret or simulate various consequences of porosity. Researchers often begin their analysis of diffusion or penetration within pores by making various simplifying assumptions, which may involve selecting an arbitrary degree of interconnectedness. As noted by Stamm (1953) a bubble of air in a pore sometimes can be just as effective in impeding flow as a physical blockage.

To predict effects of interconnectedness on diffusion of polymers in solution, Gauthier and Slater (2008) proposed a new one-dimensional lattice Monte Carlo algorithm that considered the polymer as single Brownian diffusing particle. They integrated various effects such as the entropic forces acting on the polymer segments that are outside the channel, the external forces that are pulling the polymer through the pore, and the frictional effects (inside and outside the pores) involving the polymer chains and their environment.

\section{Pinch-points}

The velocity of an incompressible Newtonian fluid passing through a smooth cylindrical pore under a specified pressure, assuming laminar flow, is proportional to the square of the radius. It follows that if a channel contains portions in which the radius is smaller, such restricted areas will have a disproportionate effect on the overall resistance to flow. For permeation in solid wood, one of the best examples of a pinch-point consists of the pit junctions that join fiber lumens (Meyer 1974). It has been shown that pretreatment with cellulase enzymes can break down the pit membranes that obstruct flow through the lumen network within wood (Jacobs-Young et al. 1998); such treatment was found to greatly increase the penetration rates of pulping liquors, especially in the direction of the fibers within a chip.

The relative permeability is even more affected in cases where the fluid contains dispersed or macromolecular substances with dimensions similar to those of the pores.

Wu et al. (2009). "Polyelectrolyte permeation in cellulose," BioResources 4(3), 1222-1262. 1227 
For example, if a polyelectrolyte molecule needs to adopt a compromised conformation (e.g. elongated or stretched) in order to pass through the pore, then one can expect an additional contribution to flow resistance (Andrews et al. 1998; Odell and Haward 2008). If a pinch-point is small enough to effectively prevent a polyelectrolyte molecular from passing through, then the molecule may have to take a more circuitous path, effectively increasing the tortuosity in terms of that substance (Olson et al. 2005).

\section{Wettability}

Wettability is the tendency for a liquid to spread on a solid surface. Relative to permeation into porous materials, such considerations can be important when there are two or more fluid phases present, e.g., polar (water), non polar (oil), or gaseous (air). For example, wettability of paper plays an important role relative to the penetration of coatings and inks (Bousfield and Karles 2004). The wettability of paper can be influenced by the fluid properties, surface forces, and complex pore geometry. Iveson et al. (2001) showed that wetting can be successfully included in a model of permeation of a liquid into initially dry porous particles that are submerged in a fluid. Factors affecting such permeation include capillary forces, viscous resistance of the liquid as it passes through narrow channels, and the pressure of any air that may be trapped within the particles. Because it may take a long time for such trapped air to dissolve into the adjacent fluid and eventually diffuse away, there can be a significant delay in achieving complete wetting. Cellulose pulp manufacturers often attempt to overcome such problems by pre-steaming of wood chips, so that the interior spaces are filled with water vapor rather than air before introduction of cooking liquors (Stamm 1953; Paranyi and Rabinovitch 1958; Malkov et al. 2003; Törnqvist et al. 2004). In principle, the same effect could be achieved by applying vacuum to wood before addition of a penetrating liquid (Stamm 1953).

The surface free energy of cellulosic surfaces can be expected to have a major impact on permeability of liquids into initially dry material. Dourado et al. (1998) showed that rates of wicking can be used as a sensitive means of determining the free energy of microcrystalline cellulose. The system's capillary force can be basically described in terms of two variables, the radius (or an equivalent radius) of the capillary, and the interfacial surface energies of the solid-liquid-gas interfaces. When the interfacial surface energies change, the contact angle (meniscus shape) and consequently the pressure difference across a liquid-gas interface will change to reestablish equilibrium.

Papermakers employ internal sizing chemicals, e.g. alkylketene dimer and rosin products, to hydrophobize many paper products, an effect that reduces water wettability of cellulosic surfaces (see Hubbe 2007). Sarmadi et al. (1993) studied the effects of fluorochemical repellant finishes on the wettability of nonwoven fabrics. Capillary flow rates were estimated from analysis of the water contact angles in combination with the fabric pore radius. These predictions were then compared with experimental results obtained from demand wettability and static absorption tests. The highest rate of water penetration was found in a fabric type that exhibited the lowest contact angle and the largest pore radius. Water repellency was found to be influenced more by the structure of a fabric than by the finish applied. Lin and Jorge (2005) modeled the wicking of liquid

Wu et al. (2009). "Polyelectrolyte permeation in cellulose," BioResources 4(3), 1222-1262. 1228 
through paperboard, taking into account various properties of both the liquid and the solid surfaces.

Ershov et al. (2003) showed that polyelectrolytes can have a subtle effect on wettability when their solutions are allowed to flow into quartz capillaries. The polyelectrolytes did not affect the surface tension at the air-liquid interface. However, large hysteresis effects were observed when comparing advancing $v$. receding contact angles; these effects were attributed to the formation of a polyelectrolyte film on the quartz surfaces.

\section{Fluid Attributes}

Properties of the fluid phase also can be expected to affect the diffusion and flow in pores. Important variables include fluid rheological behavior, fluid chemical composition, solute component dimensions, interfacial tension, wettability, and diffusion coefficients. If a fluid solution or suspension is sufficiently dilute, often one can neglect the interactions among the solute molecules or suspended particles. As the concentration becomes higher, the apparent viscosity may become a function of the shear rate and shear history. Increases in a dissolved polymer's molecular mass or concentration generally increase the apparent viscosity of the solution. The Mark-Houwink equation (Hiemenz and Lodge 2007) provides a means of predicting how these factors influence the intrinsic polymer solution viscosity and can help to elucidate their contributions to fluid diffusion in porous structure.

Flow fields can affect the conformation of an adsorbed polyelectrolyte, depending on the flow and shear rate relative to the wall of a pore (Fleer et al. 1993). Adsorbed chains can be either deformed by the fluid field (compressed against the substrate, or stretched in the flow direction) or desorbed from the substrate. This is especially important if the flow rate is sufficiently high and the polymer used has high molecular mass or is strongly swollen. Under some conditions in which adsorption is relatively weak, it is expected that polymer chains that first are stretched by a flow field would subsequently become adsorbed in higher amounts and adhere the substrate more tenaciously. Experiments have shown that hydrodynamic thickness can depend on flowrate. Similarly, it is reasonable to expect that flow-induced changes to conformation may have an effect on pore penetration.

\section{Viscosity}

One way that polyelectrolytes affect fluid flow is by raising the viscosity of a solution. Thus, Andersson and Jarnström (2006) studied the controlled penetration of starch during the surface-sizing of paper and board. The colloidal stability of surface size dispersions affected the location of components in the final sized substrate. Results showed that salt-induced flocculation lowered the penetration of amphoteric starch into the porous substrate, while the opposite effect was observed in the case of the anionic starch, probably due to screening-reduced adsorption. Viscosity was shown to affect the diffusion of sizing agents. It was found that the location of surface sizing components could be controlled by tuning of colloidal interactions in the dispersion. 


\section{Dissolved polymer attributes that can affect permeation}

The adsorbed amount of polyelectrolytes within cellulosic fibers or other porous media depends on the molecular mass of the polyelectrolyte. A decrease in molecular mass tends to increase the adsorbed amount. This rule holds true for many cationic watersoluble polyelectrolytes, such as polyethyleneimine, cationic dextran, and polydiallyldimethylammonium chloride (Wågberg 2000). By contrast, earlier work by Tatsumi and Yamauchi (1997) showed that a high-mass cationic copolymer of acrylamide remained mostly on the outer surfaces of cellulose fibers in solution. A distribution of molecular mass of the polyelectrolyte implies that some of the material can reach different sites within the fine-scale porous structure of a cellulosic substrate. However, because polyelectrolyte diffusion into porous media involves conformational changes, in addition to the strength of interaction with substrate surfaces, it is possibly misleading to attribute such effects to size considerations alone.

Important new information relative to cationic polyelectrolyte diffusion into cellulosic fibers has been provided by Horvath et al. (2008). Using fluorescent-labeled polyelectrolytes and confocal microscopy, they showed dependencies of the rate and extent of permeation on polyelectrolyte charge density and salt concentration. Highcharge-density polyelectrolytes, such as poly-diallyldimethylammonium chloride, required months to fully diffuse into the cellulose, whereas lower-charge cationic dextran molecules diffused into the cell walls in a matter of hours. Diffusion from salt-free solution did not proceed very far, appearing to be mainly restricted to the surface regions. By contrast, increased diffusion into cellulose fiber cell walls was observed with increasing salt content of the solution. The adsorbed amount increased roughly in proportion to the logarithm of elapsed time in contact with the polyelectrolyte solution.

One of the most intriguing findings of the cited work was that cationic polyelectrolytes adsorbing near to the surface of the cellulosic fibers did not appear to progress further into the cellulose with the passage of time (Horvath et al. 2008). Rather, the initial adsorbing macromolecules became more or less fixed in location, and laterarriving macromolecules had to diffuse past the already-adsorbed molecules.

Another event that can arrest the flow of polyelectrolyte-containing solution through the channels of a packed bed is the formation of polyelectrolyte complexes (De et al. 1997). The cited authors found that because the complexes required sufficient space in which to form, they tended to precipitate within relatively large pores of a packed bed, leaving the smaller channels in the bed relatively free of obstructions to flow. In this way, the formation of polyelectrolyte complexes could be used as a means of directing more of the flow towards smaller channels.

\section{Interactions that Affect Flow and Diffusion into Pores}

Three kinds of interactions between the penetrating solution and the cellulosic substrate are of prime interest. First, various interactions can affect the angle of contact between the solid and liquid phases, thus affecting the capillary pressure that often provides the main driving force for wetting to take place. Such changes in contact angle can be critical during flow into initially dry cellulose. Second, the aqueous solution may affect the swelling of the material, thus affecting pores size. And third, the solute may either be attracted or repelled from the surfaces; the strength of adsorption of solute

Wu et al. (2009). "Polyelectrolyte permeation in cellulose," BioResources 4(3), 1222-1262. 1230 
molecules onto the pore walls can be expected to affect surface charge, and in some cases adsorption can have a significant effect on the remaining open area for solution and solutes to pass through constricted areas of the pore network.

The work of Sekine and Nakatani (2002) provides a good example of how the charged nature of a solute can promote its permeation into porous material. The role of electrostatic attractions between a cationic dye and the negatively charged internal surfaces of silica gel were demonstrated by varying the $\mathrm{pH}$ and ionic strength. Greater dye permeation and sorption occurred at higher $\mathrm{pH}$, conditions that favor a stronger negative charge at the silica surface. Less permeation occurred at higher ionic strength, consistent with a weakening of electrostatic effects. Permeation was also strongly affected by the pore size of the silica gel (Sekine and Nakatani 2004). An apparently increasing diffusion constant of dye molecules was observed for increasing pore size of the substrate.

\section{Interactions that may affect pore size}

Simple ions in water can affect diffusion within cellulosic materials by affecting the swelling, and hence by changing the effective size of the pore spaces. Past studies have shown that more swollen pulps tend to be more accessible to tracer substances (Bendzalova et al. 1996). In general, relatively high levels of salt ions can be expected to decrease swelling, due to the suppression of electrostatic and osmotic forces (Lindström and Carlsson 1982; Scallan 1993). By shortening the range over which electrostatic forces of repulsion can act, the carboxyl groups that are found in many cellulosic materials become less effective in swelling the material. What is less often noted is that such salt effects can have an opposite trend at the limit of very low levels of ions in solution. Thus, relatively low levels of swelling have been observed in cases where deionized water has been used to suspend ion-free cellulosic fibers (Fält and Wågberg 2003; Li and Dai 2004). The latter observations have been attributed to a mismatch between the size of the available pore spaces and the space required for the existence of counter-ions in a diffuse double layer (Hubbe 2006).

The $\mathrm{pH}$ of the solution can be critically important to the wetting of cellulosic materials due to the fact that the water-loving character of the surfaces will be affected by the state of association of carboxylic acid groups. Thus, if the $\mathrm{pH}$ is higher than about 5 , a majority of carboxyl groups associated with the outer cellulosic surfaces can be expected to be in their negatively charged carboxylate form (Herrington and Petzold 1992). As noted in an earlier review article (Hubbe 2006), the situation can be more complicated in the case of carboxyl groups on the surfaces of very small pores within the cell walls of fibers. In such cases dissociation may be suppressed due to the lack of sufficient space for the formation of ionic double layers adjacent to a charged surface. Such effects may be equivalently viewed as a difference in $\mathrm{pH}$ between the bulk phase and within the gel structure of the cellulose (Wågberg et al. 1989; Bygrave and Englezos 1998). Such differences can be expected to become less important with increasing electrolyte concentration, since double-layer thickness becomes very small in the presence of salt ions (Hiemenz and Rajagopalan 1997). 


\section{Interaction effects of polyelectrolytes}

The degree to which a polyelectrolyte is either attracted or repelled from a substrate can have manifold consequences relative to its permeation. Before considering other, more subtle effects related to permeation, it can be useful to review how charge characteristics of a polymer and substrate are likely to affect adsorption. There often is a mechanistic relationship between a polymer's adsorption characteristics and its rate or extent of permeation.

In the case of a charged polymer one can expect mutual repulsion between the like-charged segments. This repulsion can oppose accumulation in a surface region. A surface can carry a charge as well, and it is expected that changing the environmental conditions such as $\mathrm{pH}$, salt concentration, etc., would change the number of active surface sites. In particular, an increase in salt can decrease the energy penalty resulting from charge accumulation at a surface. But at the same time, increasing salt will tend to weaken electrostatic interactions between a charged polymer segment and an oppositely charged site on a substrate.

The main effects of charge interactions within and between a polymer and a surface, including both like-charge interactions and opposite-charge interactions, are summarized in Table 1, which is adapted from Fleer et al. (1993). The table shows in a general sense how the charged nature of both a dissolved polymer and a substrate can be expected to affect adsorption, with special attention paid to the effects of salt addition. The quantity $\chi_{s}$ in Table 1 is the adsorption energy parameter, given by the difference in adsorption energy of the solvent $v s$. that of the polymer, all divided by the thermal energy $k T$. Spontaneous adsorption from the solvent onto the substrate occurs if $\chi_{\mathrm{s}}$ has a positive value (Fleer et al. 1993).

Table 1. Polyelectrolyte Adsorption as a Factor Related to Permeation

\begin{tabular}{|c|c|c|c|c|}
\hline & Polymer & Surface & Main factor(s) & Salt effect \\
\hline A & Uncharged & Uncharged & Competition between ions and polymer segments & weak \\
\hline $\mathrm{B}$ & Charged & Uncharged & Mutual repulsion between polymer segments & + \\
\hline C & \multicolumn{2}{|c|}{ Same charge signs } & $\begin{array}{l}\text { Segment-segment and segment-surface repulsion } \\
\left.\text { (high } \chi_{s}\right)\end{array}$ & + \\
\hline D & \multirow{2}{*}{\multicolumn{2}{|c|}{ Opposite charge signs }} & D1 pure electrosorption $\left(\chi_{s} \leq 0\right)$ & - \\
\hline $\begin{array}{l}D \\
2\end{array}$ & & & $\begin{array}{l}\text { D2 electrosorption enhanced by surface affinity } \\
\qquad\left(\chi_{s}>0\right)\end{array}$ & +or - \\
\hline
\end{tabular}

One of the most fascinating aspects related to Table 1 is the concept that ionic charge can affect adsorption even in cases where the water-soluble polymer is uncharged. For example, adsorption of polyethylene oxide (PEO) on silica will decrease with increasing $\mathrm{pH}$, holding other conditions constant. A more general effect has to be considered, namely, the fact that the surface charge has to be compensated by counter- 
ions in the double layer, competing for space with the uncharged segments. The competition is enhanced when counter-ions have a non-electrostatic affinity for the surface. Segment-surface interaction involving electrostatic forces can either promote or hinder the adsorption. As this kind of electrostatic forces can be screened by salt ions, it is reasonable to expect results to be affected by the type of ions, their concentrations, as wells as the $\mathrm{pH}$.

\section{MECHANISMS OF DIFFUSION AND FLOW INTO A POROUS SUBSTRATE}

The previous section highlighted examples showing the effects of different factors on the permeation of polyelectrolytes and other substances from solution into porous materials. A number of mechanisms were mentioned to account for these effects. The present section considers some of these mechanisms in greater detail. By applying such concepts, permeation phenomena can be understood as an overlapping set of thermodynamic, kinetic, substrate morphological, and mechanical considerations that together govern the location of a polyelectrolyte as a function of time.

The fluid phase will be considered first. The fluid system may be a single phase, such as pure water, an aqueous solution, or multi-phase (such as water with air or oil, or others). In the case of multiple phases, the fluids might be immiscible or miscible. Solutes can significantly affect flow behavior. The discussion below will start with simple cases and works toward more complicated cases.

\section{Pure Water}

The movement of pure water into porous solids, including cellulosic materials, can serve as a kind of introduction to the mechanisms controlling permeation. Water's permeation into porous solids can be expected to occur by means of diffusion and/or flow.

\section{Diffusion (ordinary and anomalous)}

Though many details of diffusion theory are beyond the scope of this article, some general concepts can be summarized. Diffusion phenomena generally obey Fick's first law (1855), which for the flux of a selected substance in an isotropic material can be written as,

$$
J_{x}=-D(\partial C / \partial x)
$$

where $D$ is the diffusivity constant, $C$ is the concentration or number density, and $x$ is the distance in the direction perpendicular to the gradient of concentration. This relationship follows from a "random walk" analysis of motions at the level of individual particles or molecules undergoing the diffusion process. The diffusion constant is generally related to the thermal energy of the system (i.e. the temperature), the viscosity of the fluid, and the size of the particle. In the case that a diffusing particle can be described as a sphere, its diffusivity is given by the Stokes-Einstein relation, 


$$
D=k T /[6 \pi \eta r]
$$

where $k$ is the Boltzmann constant, $T$ is the absolute temperature, $\eta$ is the solution viscosity, and $r$ is the particle's radius.

One of the most immediate consequences of Equation (2) is that the rate of diffusion increases with increasing temperature. This is a consequence not only of the higher kinetic energy within the system, but also due to a decreasing fluid viscosity with increasing temperature. Robertson and Lönnberg (1991) observed, however, that rates of diffusion of solutes in wood increased with temperature according to an Arrhenius relationship, suggesting that the rate-limiting step involved overcoming of an energy barrier (see also Stamm 1959).

Li et al. (1992) considered the self-diffusion of water molecules within neverdried bleached softwood kraft pulp fibers. Using a pulsed echo spin gradient (PGSE) nuclear magnetic resonance (NMR) method, they detected two echo attenuation profiles associated with water in the pores. One of these was attributed to water between the fibers, and the other was associated with water within the cell walls. The self-diffusion coefficient of water was reduced slightly even in the relatively large spaces between the fibers. This effect was attributed to the obstructing effect of cellulosic material. VargasFlorencia et al. (2007) came to a similar conclusion in their study of water within nanostructured $\mathrm{TiO}_{2}$ films, and Veith et al. (2004) found restricted diffusion in the case of water within silica gel. Within the cell walls Li et al. (1992) observed that the selfdiffusion of water was reduced by approximately one-third, in comparison to bulk water. Greater diffusion was detected in the length-wise direction of fibers, an observation that is consistent with the expected orientation of pores. It was concluded that pores within the walls of the fibers had lengths in the range of about 2-20 $\mu \mathrm{m}$, but that they were quite narrow in other dimensions. Ek et al. (1994) used a similar approach to characterize the pore sizes within cellulose beads of differing porosity.

The term "anomalous diffusion" has been used to describe the mobility of water that is very close to a solid surface. Mitra et al. (2001) employed quasi-elastic neutron scattering to distinguish between different types of water within porous alumina having a mean pore size of $5 \mathrm{~nm}$. Some of the water appeared to be essentially bound to the solid surfaces. Other water displayed restricted diffusion. Ironically, the results showed higher self-diffusion rates of the non-attached water within small pores at temperatures near of below the freezing point of bulk water, suggesting that freezing did not occur within the pores of alumina under the conditions of testing. In other words, the water within the tiny pores appeared to be super-cooled liquid below the freezing point of bulk water. These ideas are consistent with the findings of Weise et al. (1996) and Maloney et al. (1998), who used differential scanning calorimetry to quantify the amounts of bulklike water, freezing bound water, and non-freezing bound water within the small pores of fiber cell walls. Nakashima (2003), who used a spin-echo type NMR method, reported similar observations in the case of clay suspensions of the type that delaminate to a great extent in suspension. Topgaard and Soderman (2001), who used a similar approach to study wetted paper, obtained information about the pore structure within fibers. Tovbin (2005) carried out numerical simulations that helped to support the interpretation of data from these types of observations. Likewise Kim et al. (2005) concluded, based on 
numerical simulations, that simple fluids would experience restricted diffusion when the pore size was less than twice the diameter of the diffusing molecules.

\section{Viscous flow}

In the simplest case of an incompressible Newtonian liquid entering a smooth, cylindrical pore under laminar flow conditions, the average fluid velocity can be estimated by considering the contributing effects of viscous resistance to flow, capillary pressure, and any externally applied pressure. For a cylindrical capillary the viscous resistance to flow can be expressed in terms of a pressure as,

$$
\Delta P_{\text {viscous }}=8 \eta v L / R^{2}
$$

where $\eta$ is the viscosity of the fluid, $v$ is the average velocity in the pore, $L$ is the wetted length of pore, and $R$ is the pore's radius. The capillary pressure is given by

$$
\Delta P_{\text {capillary }}=2 \gamma \cos \theta / R \text {, }
$$

where $\gamma$ is the tension at the air-liquid interface and $\theta$ is the angle of contact between the liquid and the solid (measured through the liquid phase). In cases where only capillary and viscous forces are opposing each other, these two expressions can be set equal to each other, yielding the following expression,

$$
\mathrm{d} L / \mathrm{d} t=\gamma R \cos \theta /\left[\begin{array}{lll}
4 & L
\end{array}\right]
$$

where $L$ is the distance that the liquid already has penetrated into the pore and $t$ is time. Equation 5 then can be integrated to give the well-known Lucas-Washburn equation,

$$
L=\left\{\left(2 r \gamma_{\mathrm{LV}} \cos \theta t /(4 \eta)\right\}^{1 / 2}\right.
$$

Flow also can be influenced by a pressure differential between the inside and the outside of a porous material. The following equation can be used to estimate the extent of fluid penetration as a function of time under the combined influences of capillary wetting and applied pressure (see Eklund and Salminen 1987),

$$
L=\left\{\left[\left(2 r \gamma_{\mathrm{LV}} \cos \theta+\Delta P_{\text {appl. }} r^{2}\right] t /(4 \eta)\right\}^{1 / 2}\right.
$$

where $\Delta P_{\text {appl }}$ is the applied pressure difference between the liquid phase and the interior of a porous material.

Because the foregoing equations were based on simple, cylindrical pores, one would not necessarily expect that they could be applied to real cellulosic materials. Nevertheless, the square-root dependency of penetration depth vs. time, as predicted by Eq. 6, has been observed in the case of low-surface-energy fluids penetrating into paper (Bristow 1967; Lyne and Huang 1993). Deviations from that relationship have been attributed to surface roughness of paper and to delayed wetting of cellulosic surfaces due to gradual rehydration (Lyne and Aspler 1982). In addition, it has been shown that the 
liquid-gas interfacial tension term $\gamma_{L V}$ can be strongly time-dependent; thus, values of $\gamma_{L V}$ determined under quasi-static conditions, as with a Denouy tensometer, are not necessarily expected to give accurate predictions for rapid wetting phenomena, as occur during lithographic printing (Aspler et al. 1987). The discrepancy was attributed to the fact that surfactant molecules may require time to migrate to an interface, especially if the interfacial area is being created at a relatively rapid rate.

In the preceding equations it is implicitly assumed that the liquid cannot slip at the fluid-solid interfaces. However, it can be worth examining this assumption in specific instances, such as cases involving very small pores (Dimitrov et al. 2007). Thus Saugey et al. (2005) carried out a numerical study of liquid confined between two planes, and they varied the boundary conditions. Substantially higher apparent diffusion constants can be expected if the conventional boundary assumptions are replaced by a "partial slip" condition. It was proposed that their calculated results could be used to establish whether such boundary conditions provide a useful description of any real systems.

\section{Capillary forces (and geometrical effects)}

Interfacial tension is the amount of work per unit area that has to be done in order to separate one phase, say a liquid, from another phase, say, a solid, gas, or another immiscible liquid. Interfacial tension comes from the inward attraction of the molecules towards the interior of a substance upon those on the surface. The work required to separate a unit area of a solid from a liquid is related to the corresponding surface and interfacial tensions $\gamma$ by,

$$
W_{\mathrm{SL}}=\gamma_{\mathrm{S}}+\gamma_{\mathrm{L}}-\gamma_{\mathrm{SL}}
$$

where the subscripts refer to the solid-gas, liquid-gas, and solid-liquid interfaces.

Capillary forces resulting from interfacial tensions may cause a fluid to penetrate into small spaces that are filled with another liquid or air. As discussed by Senden et al. (2000), significant research in this area started in the 1950s. Calderwood and Mardles (1955) studied the rate of flow of liquids into capillaries. Tollenaar (1954) investigated the rise of liquids into a wedge-shaped crevice.

The geometries of pores can profoundly affect the capillary wetting of pores. Wetting tests even can be used to evaluate the geometries of pores. Thus, Everett et al. (1977), who used a simple gravimetric technique to determine the rate of capillary imbibition into glass-fiber filter papers, were able to use the Lucas-Washburn equation (Eq. 6) to obtain an "imbibition-equivalent" pore size. Although it is difficult to relate pore sizes measured in this way to the real geometry of a fiber mat, it would nevertheless be valuable to seek empirical correlations to such variables as fiber diameter and packing density. Pre-wetting of the porous substrate was found to decrease the rate constant for subsequent imbibition. Bousfield and Karles (2004) likewise were able to use simplifying assumptions to model the capillary penetration of printing ink vehicle and related fluids into porous coatings on the paper surface. 


\section{Thermal transport of water through cellulosic material}

Temperature gradients within wood or other porous material can be expected to result in gradients of moisture content. The mechanism appears to involve preferential evaporation from hotter locations and preferential recondensation in cooler locations (Skaar and Siau 1981). This kind of effect can be a nuisance in various practical applications, such as in the storage of paper, where the outside of a paper roll or stack tends to cool first, resulting in a higher moisture content toward the outside. A baggy or puckered appearance of the outside can result due to the greater swelling in locations having higher moisture content. A related mechanism can cause migration of wood extractives towards the outer surfaces of paper rolls or stacks as they cool; this situation can result in greater hydrophobicity ("self-sizing") in those locations (Swanson and Cordingly 1959).

\section{Molecules in Solution}

Another layer of complexity needs to be considered when the liquid phase contains a solute, even in cases where the solute molecules are very small relative to the size of pores. For instance, many researchers have studied the diffusion of metal ions in cellulosic (Cooper 1998; Ra et al. 2001, 2002; Davis and Van Hise 2006; Srivastava et al. 2006) and other porous materials (Osaki et al. 1993; Kunetz and Hench 1998; Tantemsapya and Meegoda 2004; Fisher and Eizenberg 2008). In bulk solution the diffusion of ions in aqueous solution can be expected to be proportional to a concentration gradient; in other words, rates of diffusion ought to obey Eq. (1). Diffusion generally can be explained in terms of a "random walk" process, which over the passage of time would be expected to yield a more uniform concentration.

\section{Ion exchange}

Though it would make logical sense to assume that equations (1) and (2) could be used to predict rates of metal ion diffusion, relative to a concentration gradient into cellulosic material, various studies have shown instead that the rates of diffusion and uptake in such cases may be controlled by ion exchange effects (Osaki et al. 1993; Cooper 1998; Kunetz and Hench 1998; Srivastava et al. 2006; Sud et al. 2008). In other words, the metal ions are at any moment partitioned between the liquid phase and an adsorbed state. In the case of cellulosic materials it would be expected that metal ions would associate themselves with bound carboxylate groups. The ion exchange capacities of cellulosic materials have been found to vary within wide limits, depending on conditions of preparation and purification (Herrington and Petzold 1992).

Osaki et al. (1993) found that the diffusion coefficients of $\mathrm{Sr}$ and $\mathrm{Co}$ in silica gel layers decreased with the second power of porosity. If the porosity was kept constant, the diffusion coefficients depended on the distribution coefficients $(K)$ of $\mathrm{Sr}$ and Co between silica gel and water in pores as a function of $1 /(1+K)$ at various pHs. Kanungo and Collinson (2003) observed that the diffusion rates of some ions were the same in silica gel medium, compared to the bulk solution, whereas other ions displayed sharply reduced diffusion rates in the gel. The latter effect was attributed to relatively strong molecular interactions. 
Cooper (1998) found that the diffusion coefficients of copper in wood samples were about $1 / 10$ to $1 / 10,000$ times those of water molecules, depending on $\mathrm{pH}$ and temperature conditions. Durr et al. (2005) studied the adsorption of ruthenium-dye molecules out of ethanol solution onto and through nanoporous $\mathrm{TiO}_{2}$ films. They found these diffusion constants through the fine porous network were up to three orders of magnitude lower than in bulk ethanol; the results were discussed with respect to the material properties and pore structrure.

Suppressed diffusion of solute molecules within very small capillaries also can be predicted based on molecular dynamics simulations. Thus, Lai et al. (2004) predicted that electrolytes would have a slower diffusion rate by as much as two orders of magnitude within a fine porous material.

\section{Surfactant Solutions}

Surfactants can be expected to have an especially large effect on transport into or through porous materials in cases involving more than one fluid phase, e.g. water and air or water and oil. However, it is not always legitimate to expect well-established laws governing macroscopic aspects of capillarity to remain true for interactions at the submicroscopic level. Thus Starov et al. (2004) considered spreading of small liquid drops over thin dry porous layers. They proposed that drop motion over a porous layer is caused by interplay between the two processes of spreading (expanding of the drop base) and imbibition (drop shrinkage during expansion of a wetted region inside the porous layer). If the mean pore size was below a critical value, then the permeability of the porous medium was not influenced by the presence of surfactants, whatever the value of the concentration. If the mean pore size was larger than the critical value, then the permeability increased with increasing surfactant concentration. Such results suggest that surface-active agents require a certain minimum of space in order to exhibit the kinds of effects that can be observed as a macroscopic scale.

Permeation of the surfactants themselves also can be expected to be profoundly affected by their adsorption onto the walls of porous materials. Petel'skii and Abramzon (1999) studied the process of dialysis of solutions of surfactants through porous membranes. They found a dependency of surfactant molecule size vs. permeation rate. Physical adsorption of surfactants on the pore walls during the dialysis decreased the membrane permeability. It was proposed that the sorption of the diffusing substances largely depends on van der Waals interactions instead of electrostatic forces.

\section{Surface diffusion}

In cases where solute molecules are attracted to pore wall surfaces it is reasonable to consider whether such solute material can migrate while in contact with the surface (Alanissila and Ying 1992; Ho et al. 2000; Choi et al. 2001). As a prime example, it is reasonable to expect cellulase molecules to diffuse along cellulosic surfaces (Jervis et al. 1997). Such "surface diffusion" also has been proposed to explain rates of permeation of direct dyes into water-swollen cellulose membranes (Gutsche and Yoshida 1994; Maekawa et al. 1995). 


\section{Emulsification}

Surfactants can be expected to dramatically affect the transport of various other solute molecules in cases where the latter can be emulsified or stabilized. This is one of the underlying mechanisms of detergency (Ang and Abdul 1991). Thus, Weigenand et al. (2007) found that surfactant was beneficial for the permeation of silicone into wood. Microemulsions were much more effective penetrants, in comparison to emulsions having a typical droplet size. Surfactants also have been found effective for enhanced oil recovery (El-Batanoney et al. 1999; Summ and Soboleva 2003; Standnes 2004). Ang and Abdul (1991) used an alcohol ethoxylate surfactant to wash automatic transmission fluid from sandy soil. Water washed little of the oily matter from a column of the soil, while dilute surfactant solutions washed the soil much more effectively. Displacement of oil was proposed as the main washing mechanism. Supplementary mechanisms of oil removal were dispersion of oil (due to reduced surface tension), and the solubilization of oil by surfactant micelles.

Vesicles, which can be described as droplets defined by surfactant bilayers, can serve as models for biological cells. Linke et al. (2006) carried out a theoretical study of their passage through fine pores. Rates of passage were predicted based on the thermodynamic fluctuations of the membrane shape, and also the requirement of water to pass through the bilayer in order for the vesicle to be small enough to get past narrow points in the pore.

\section{Multiphase flow in porous media}

Complex interactions take place if an immiscible mixture is forced through an array of capillaries. As a general rule, a given channel can be expected to contain a single fluid phase, and other components of an immiscible mixture will generally be found in other channels. The mixing of miscible liquids in capillary networks also has been considered (Levenspiel and Smith 1957; Levenspiel 1958); though the details of mixing present computational challenges even in that case, diffusion processes ensure that the system tends toward a uniform mixture with the passage of time.

\section{Polyelectrolytes in Solution}

In comparison to the simple ions and surfactants just considered, polyelectrolytes can be described as relatively large, deformable, and possessing a multiplicity of charged groups. All of these factors can be expected to influence their diffusion, as well as their flow behavior in pores.

\section{Wettability effects}

It is reasonable to expect that the presence of polyelectrolyte in a fluid ought to affect wetting and capillary imbibition phenomena. Ershov et al. (2003) found such evidence when they measured the flow rates and the dynamic contact angles of aqueous solutions of a cationic polyelectrolyte in quartz capillaries. Their results showed that the macromolecules adsorbed on the negatively charged quartz surface, resulting in a change of contact angle. As the solution concentration increased, the advancing contact angles were reduced, favoring wetting. 
Though dissolved polyelectrolytes can be expected to increase the viscosity of a solution, it can be misleading to employ shear viscosity information to predict resistance to flow through porous media. That is because the presence of high-mass dissolved polymers can greatly increase the extensional viscosity of the solution, leading to substantial resistance to flow at the entrances and exits of pores, as well as anywhere in a network of pores where a flow channel narrows or widens (Andrews et al. 1998; Odell and Haward 2008). As noted by Shaqfeh and Kock (1992), dissolved polymers can be expected to become stretched if they are present in a solution that is forced through a packed bed. Such flow conditions also can lead to the break-down of high-mass polymers in solution (Nguyen and Kausch 1992). In related research, Carrington et al. (1996) used extensional flow measurements to find evidence of intermolecular effects affecting the flow behavior of xanthan gum solutions.

\section{Molecular mass}

Effects of molecular mass on the adsorption of polyelectrolytes onto cellulose and other porous materials have been shown in various studies. In contrast to what would be expected in the case of non-porous surfaces, many studies have shown increasing adsorbed amount with deceasing molecular mass of the polyelectrolyte (Hostetler and Swanson 1974; Petlicki and van de Ven 1994; Gruber et al. 1996; Alince and van de Ven 1997; Hubbe et al. 2007b; Wang et al. 2007). Petlicki and van de Ven (1994) investigated the adsorption of a low and a high molecular weight polyethyleneimine (PEI) onto pulp fibers. They found that low molecular weight PEI could penetrate into the porous wall of the fibers, while for high molecular weight PEI, adsorption in pores was negligible. Evidence of much slower penetration of polyelectrolytes, with increasing mass, has been found in studies that employed electrokinetic measurements (Koethe and Scott 1993; Farley 1997; Wang and Hubbe 2002; Hubbe et al. 2007b; Wu et al. 2008). By contrast, Jeremic et al. (2007) observed that poly(ethylene glycol) penetrated the cell walls of red pine wood without any obvious effects of molecular mass in the relatively low range of 1000 to 20,000 Daltons.

\section{Charge density}

There are several reasons to expect the charge density of a polyelectrolyte to affect its transport within fine pores of wet cellulosic material. First, as already discussed, ionic charges on a dissolved polymer cause it to adopt a more expanded molecular conformation. But in addition, one can expect interactions with charged groups on and within pores of the substrate should become stronger with increasing charge density of the polyelectrolyte. The adsorption of polyelectrolyte on an oppositely charged substrate can be considered to be a form of polyelectrolyte complexation, and such interactions become stronger with increasing charge densities (Fleer et al. 1993). Such an effect may at least partly explain why Horvath et al. (2008) observed very slow adsorption of high-charge-density cationic polymers into cellulose, in comparison to lower-charge-density polyelectrolytes. If a polyelectrolyte molecule becomes strongly adsorbed and essentially immobilized in the entrance region of a porous system, then it is reasonable to expect that like-charged polyelectrolytes will experience electrostatic forces of repulsion and be less likely to diffuse into such pores. However, as was actually

Wu et al. (2009). "Polyelectrolyte permeation in cellulose," BioResources 4(3), 1222-1262. 1240 
observed in the cited study, diffusion past or through the obstructing polyelectrolytes would be expected to be facilitated by the presence of salt ions, which would tend to screen the electrostatic effects. Kim and Anderson (1991) and Linden et al. (1999) earlier considered the degree to which adsorbed polyelectrolyte may hinder the diffusion and flow of solvents and solutes, including macromolecules; Idol and Anderson (1986) showed already that adsorbed polyelectrolytes can hinder solvent convection and diffusion.

Because adsorption phenomena are often controlled by a pairing of charges on the substrate with those on an oppositely charged absorbing species, it is usual to expect increasing adsorbed amounts with decreasing charge density of the polyelectrolyte (Fleer et al. 1993; Wågberg 2000). Results of work by Wang et al. 2007 showed that the same trends remain true in the case of highly porous material. They used changes in $\mathrm{pH}$ to adjust the effective charge density of poly(acrylic acid) adsorbing from $\mathrm{NaCl}$ solution onto cationized mesoporous silica particles. The amount of polyelectrolyte adsorbed fell with increasing $\mathrm{pH}$ (and charge density) in the range 3 to 7, consistent with increasing dissociation of the carboxyl groups. In the case of the highest-mass polyelectrolyte tested, highest adsorbed amounts were obtained at an intermediate salt concentration; such results are consistent with a balance between (a) increasing salt tending to make the conformation of the polyelectrolyte less extended, allowing more to pack within a given space, and (b) increasing salt tending to weaken the charge interaction that favors adsorption (Fleer et al. 1993).

\section{Flexibility and conformation of macromolecules}

The deformability, in conjunction with thermal energy and the associated collisions of polymer segments with solvent molecules, will give rise to ceaseless changes in macromolecular conformation. To a first approximation, the "size" of a dissolved polymer can be equated to the average radius of gyration, or some other suitable measure of its most frequent dimensions that arise from the random motions. Thus it is possible to use polyelectrolytes of well-defined degree of polymerization as probes to evaluate pore sizes. Notably, Stone and Scallan (1968) used such an approach in their evaluation of pore sizes within cellulosic fibers; they chose to use uncharged, highly water-soluble dextran macromolecules in their work. It was assumed that the macromolecules were neither attracted nor repelled from the cellulosic surfaces.

Polyelectrolytes, due to their ionized groups, can be expected to have more expanded macromolecular conformations, compared to their uncharged counterparts. As noted by Horvath et al. (2008), increasing charge density of a polyelectrolyte can be expected to increase its stiffness. This expansion tends to be reduced with increasing electrolyte concentrations (Beer et al. 1997; Lin and Cheng 2000); the effect is consistent with a decrease in the Debye-Hückel length with increasing ionic strength. What is less certain is whether such considerations will still apply when a polyelectrolyte is interacting with pore walls that have charged groups of the opposite sign. In that case it may be more reasonable to expect counter-ions to diffuse away from the site of adsorption. A possible alternative contributing explanation for why Horvath et al. (2008) observed extremely slow permeation of a high-charge cationic polymer into cellulose is

Wu et al. (2009). "Polyelectrolyte permeation in cellulose," BioResources 4(3), 1222-1262. 1241 
that they employed poly-diallyldimethylammonium chloride, which is known to have a relatively stiff molecular structure (Brand et al. 1997).

The average or characteristic size of a polyelectrolyte in solution also is a key factor in determining the distribution of molecular mass, using gel permeation chromatography (GPC). As noted by Potchka (1993) one can assume that convection is the main mechanism by which polyelectrolytes progress through a GPC column, whereas diffusion will be the main mechanism by which they enter or leave a generally "stagnant zone" within the porous packing material. Reeder et al. (2000) considered an analogous system in which the porous material contains interconnected pores having a wide range of sizes; possibly this kind of modeling would also be appropriate for wood-based porous materials, including carbon black. Neuman and Walker (1992), whose analysis was not limited to polymeric solutes, showed that a mathematical model combining both mass transfer and pore diffusion gave the best predictions for packed columns of relatively large cellulosic particles, whereas simpler models were adequate in some other cases.

\section{Excluded volume}

Excluded volume is a term that is used to account for the fact that the average concentration of polymer segments at a specific location can be less than what would be calculated based on a completely random distribution of matter within a fluid phase. One type of excluded volume is due to the fact that one part of a long chain molecule cannot occupy space that is already occupied by another part of the same molecule. Such excluded volume causes the ends of a polymer chain in a solution to be further apart (on average) than they would be were there no excluded volume. This is an important factor in analyzing rates of polymer molecule penetration in porous media.

A more important kind of exclusion volume, in terms of the present discussion, involves a depletion of polyelectrolyte segments in the close neighborhood of a noninteracting surface (Fleer et al. 1993). As illustrated in Fig. 1, the origin of such excluded volume can be understood by considering the shape of a polyelectrolyte in solution as a round ball, consistent with its time-averaged segment density in space relative to its center. The substrate surface is modeled as a smooth, flat wall.
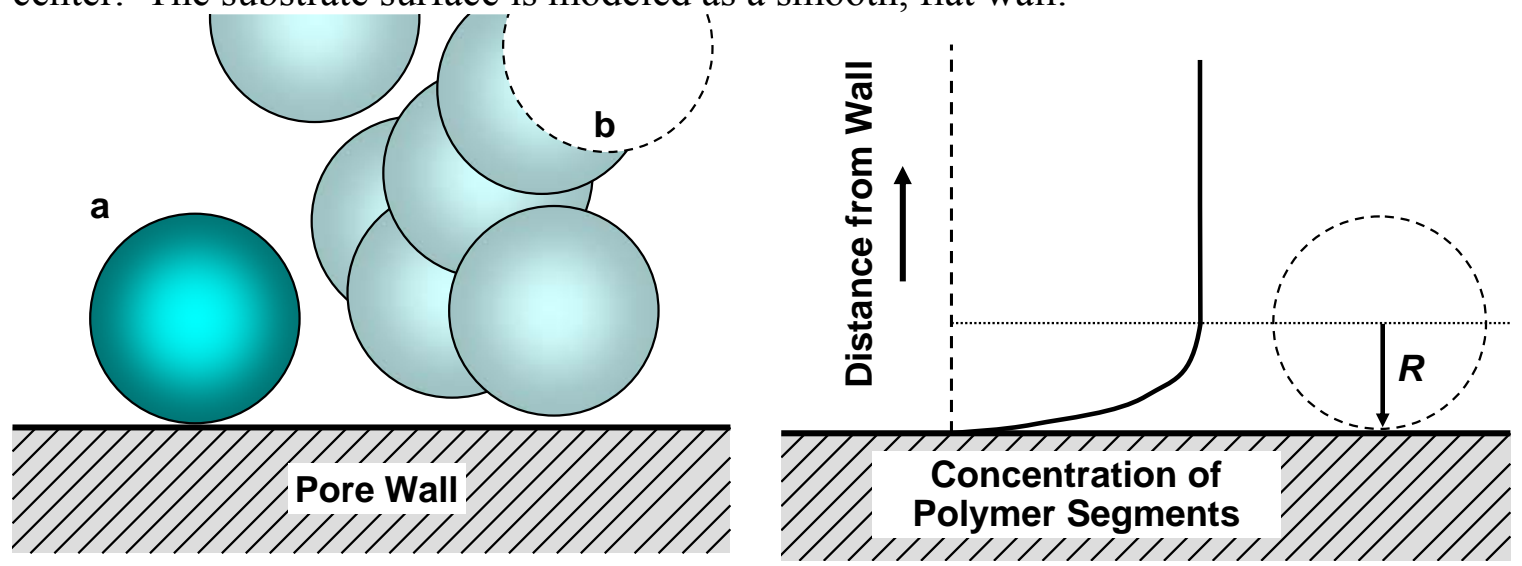

Fig. 1. Illustration of the concept of excluded volume. Left: Dissolved polymers modeled as spheres, the centers of which cannot approach closer than one radial length from a smooth wall, but which otherwise are uniformly distributed in the solution. Right: Example of an expected concentration distribution of polymer segments (for instance, if the spheres are taken as being of uniform density of polymer segments). 
The concentration of polymer segments in the bulk of solution then can be estimated in a simple way, based on the volume fraction occupied by the spheres, relative to the solution as a whole. But locations very close to the wall can be occupied only by polyelectrolyte segments that are near to the outer extremities of polyelectrolyte macromolecules, since, according to the model, the centers of the molecules are held away from the wall. It follows that there will be a reduced concentration of macromolecule segments at locations less than one radial length of the macromolecule from the wall. As noted by Alince and van de Ven (1997), this kind of effect implies that the concentration of non-interacting polymer within a sufficiently small pore is likely to be lower in comparison to the bulk concentration of the polymer.

Significant implications of excluded volumes can arise in cases where entrance of a polyelectrolyte into a fine pore changes the entropy of the system (Miklavic 1993; Kumar and Muthukumar 2008). Stone and Scallan (1968) assumed that the size of the pores can be equated to the size of non-interacting macromolecules that can fit into them. However, a polymer in a small pore, even if it has no significant interaction with the wall material, has less degrees of freedom of motion. Any macromolecular conformation that requires polymer segments to pierce the wall material has to be excluded from the physically possible set. In a real system one cannot usually expect to have enough information to accurately estimate the ratio of concentrations in the pores versus an adjacent bulk solution. The assumption of true non-interactivity also may be subject to doubt in a given case. To avoid problems related to excluded volumes and questions about affinity, Alince and van de Ven (1997) advocated the use of cationic macromolecules of different size in their analysis of pore sizes within water-swollen cellulose, which has negatively charged surfaces.

\section{Reptation}

Reptation is a term sometimes used to describe the manner in which a flexible polymer can pass through a pore space that might be too narrow to allow passage of the polymer in its average bulk-phase conformation (de Gennes 1971). As described by Teraoka et al. (1992), if a macromolecule's persistence length is greater than the characteristic size of the pores, then the macromolecule still might be expected to progress through the porous material by means of wiggling motions. The effect is illustrated in Fig. 2.

Cule and Hwa (1998) predicted that rates of reptational migration can be critically dependent on polymer structure; in the cases that they considered, a copolymer was predicted to migrate much more slowly through narrow pores in comparison to a homopolymer of similar mass. Wolterink et al. (2006) used Monte Carlo simulations to study the effects of polymer length, radius of gyration, and pore diameter on rates of translocation. Wang and Luo (2007) simulated the movement of polymer chains through a small hole with the dynamic Monte Carlo method. They studied the dependence of the relaxation time and escaping time on the chain concentration. Inter-chain interactions appeared to play an important role in the translocation process. 

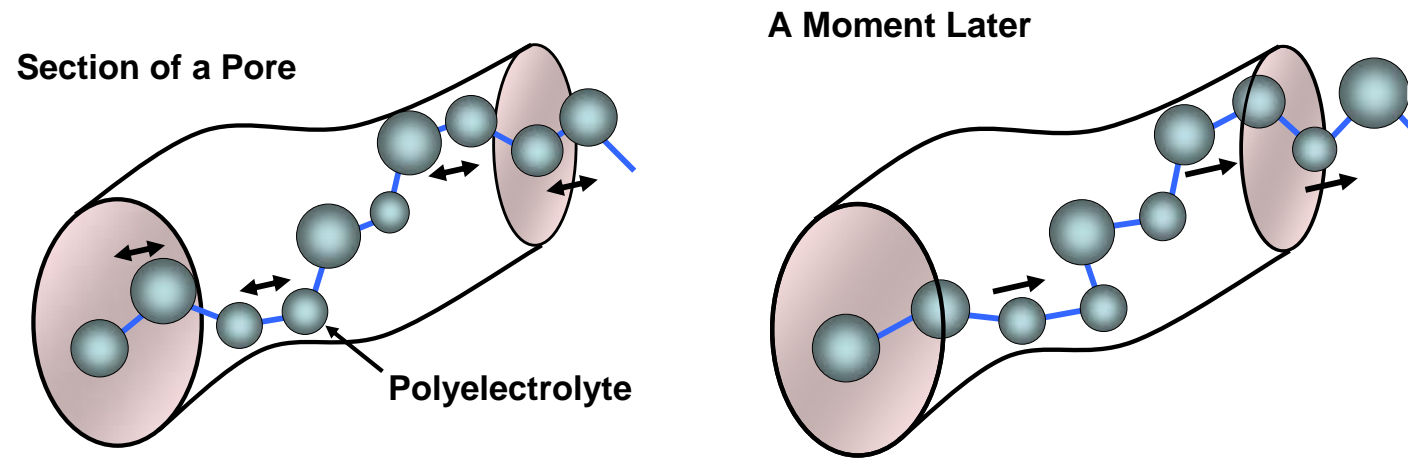

Fig. 2. Schematic illustration of reptation, a mechanism by which a solubilized polymer chain can crawl through tortuous pore passages, even if the pore size is smaller than the polymer's persistence length.

Guo et al. (1990) used quasi-elastic light scattering and observed two time scales associated with the permeation of polystyrene into porous glass saturated with toluene solution. The faster time scale was attributed to initial diffusion into the entrances of pores under conditions governed mainly by hydrodynamic interactions. The slower time scale was attributed to diffusion within the tortuous passages of the material. In general, as shown by the research group's earlier work (Bishop et al. 1986), the diffusion rate was significantly reduced relative to bulk diffusion. Lua and Grosberg (2005) modeled the migration of dissolved polymers within pores having a "sawtooth" distribution of potential energies and on this basis predicted that the time of passage may depend on the direction of diffusion.

Kabanov et al. (1989) suggested a mechanism in which polyelectrolyte molecules may follow each other in single file as they progress down especially narrow pores in a substrate. Horvath et al. (2008) did not think that such a model applied to their systems, since they observed that polyelectrolytes adsorbing near to the surface of cellulosic fibers remained pretty much fixed in location; polyelectrolyte molecules penetrating further into the fibers had to diffuse past the initially adsorbed molecules. The expected repulsion between an adsorbed cationic polyelectrolyte and another such molecule trying to squeeze past it may well explain the effect of salt concentration in the cited work (Horvath et al. 2008). In the absence of salt the effective distance over which electrostatic forces can be effective may be several nanometers, and as a consequence the initially adsorbed polyelectrolyte molecules could block further permeation. In the case of lower-charge polyelectrolytes it is reasonable to expect that such blocking effects would be less effective. Miller and Bruening (2005) considered effects of more drastic treatment of a system with alternating layers of cationic and anionic polyelectrolytes, forming a multilayer film on a porous support; in that case it was possible to control permeability of the film by adjustments in the film components. Results were well correlated to the degree of swelling in the polyelectrolyte multilayer films.

\section{Colloidal Particles in Suspension}

Many of the same factors that affect ionic solutes, surfactants, and polyelectrolytes in solution are also expected to affect the permeation of colloidal particles. This 
section with highlight some special circumstances that are likely to be important for colloidal particle permeation.

\section{Sieving}

Compared to molecular substances in water, colloidal particles are much more likely to become mechanically entrapped of "sieved." A recent review article (Hubbe et al. 2009) cited evidence that sieving is a leading cause of permeability reduction in porous media. Various publications have reported that sieving mechanisms may slow down the permeation of colloidal matter into porous materials (Inglezakis et al. 1999; Oswald and Ibaraki 2001; Yamamoto et al. 2004; Auset et al. 2005; Grznarova et al. 2005; Mays and Hunt 2005; Bauer and Nadler 2006; Shin 2006; Alvarez et al. 2007; Johnson et al. 2007; Bauer et al. 2008; Shen et al. 2008).

\section{Brownian motion of particles}

The same effect of thermal energy underlying the previously mentioned diffusion of ions and polyelectrolytes also causes very small suspended particles to undergo ceaseless semi-random motion (Einstein 1905). In the case of colloidal particles, however, such motions often can be seen through the microscope (Brown 1828). Various researchers have used calculations to predict effects of Brownian motion as a colloidal suspension passes through a porous medium (James and Chrysikopoulos 1999, 2000; Dudko et al. 2005; Chavez-Rojo et al. 2008). Nitsche and Balgi (1994) predicted that Brownian diffusion within small pores should be significantly decreased by boundary effects. In essence, the viscous resistance to particle motion becomes greater when it is very close to a wall, and such locations become dominant as pore size becomes smaller. Wei et al. (2000) and Nelissen et al. (2007) considered the special circumstance in which the pores are not large enough for two suspended particles to pass each other; kinetic relationships were therefore derived under the assumption of single-file diffusion. Ten Bosch (2001) carried out detailed Brownian dynamics calculations leading to the conclusion that colloidal particles will tend to be more concentrated near the centers of pores, assuming that there are no significant forces of attraction with the pore walls. Chaplain and Mills (1992) carried out Brownian simulations to predict the movement of colloidal particles in groundwater; their results showed that two widely different outcomes are likely. On the one hand, the rate of diffusion of an individual particle in a porous medium can be more rapid in comparison to its diffusion in the bulk solution. On the other hand, some particles may spend an extremely long time in the porous medium before they diffuse out.

\section{Electrostatic and van der Waals forces}

A more complete analysis based on Brownian motion needs to take into account electrostatic, dispersion, and hydrodynamic forces acting upon a diffusing particle. Revil (1999) and Chavez-Rojo et al. (2008) constructed models to consider all of these influences. Their calculations were found to agree with published data. Chen (1998) predicted that attractive interactive forces with pore walls will tend to slow down the diffusion of Brownian particles in comparison to their bulk diffusion, whereas the opposite should be true in the case of repulsion from the wall. These tendencies were

Wu et al. (2009). "Polyelectrolyte permeation in cellulose," BioResources 4(3), 1222-1262. 1245 
consistent with an expected enhancement of viscous resistance to motion in the case of particles near to a wall (Pawar and Anderson 1993; Dechadilok and Deen 2006).

Observations by Kluijtmans et al. (1998) provide corroboration for this work, since they observed higher diffusion rates in the case of like-charged particles, compared to uncharged particles diffusing in the same-charged porous media. In both cases the diffusion rates were much lower than in the bulk solution. The principle underlying this effect is illustrated in Fig. 3. The reason that the particle concentration profile marked " $d$ " in the figure shows depletion very near to the wall is due to the hydrodynamic effect described by Saffman (1965). In all cases Brownian motion can be expected to make the distribution of particles somewhat more uniform, so that the particles are not all focused at the position of minimum interactive energy.

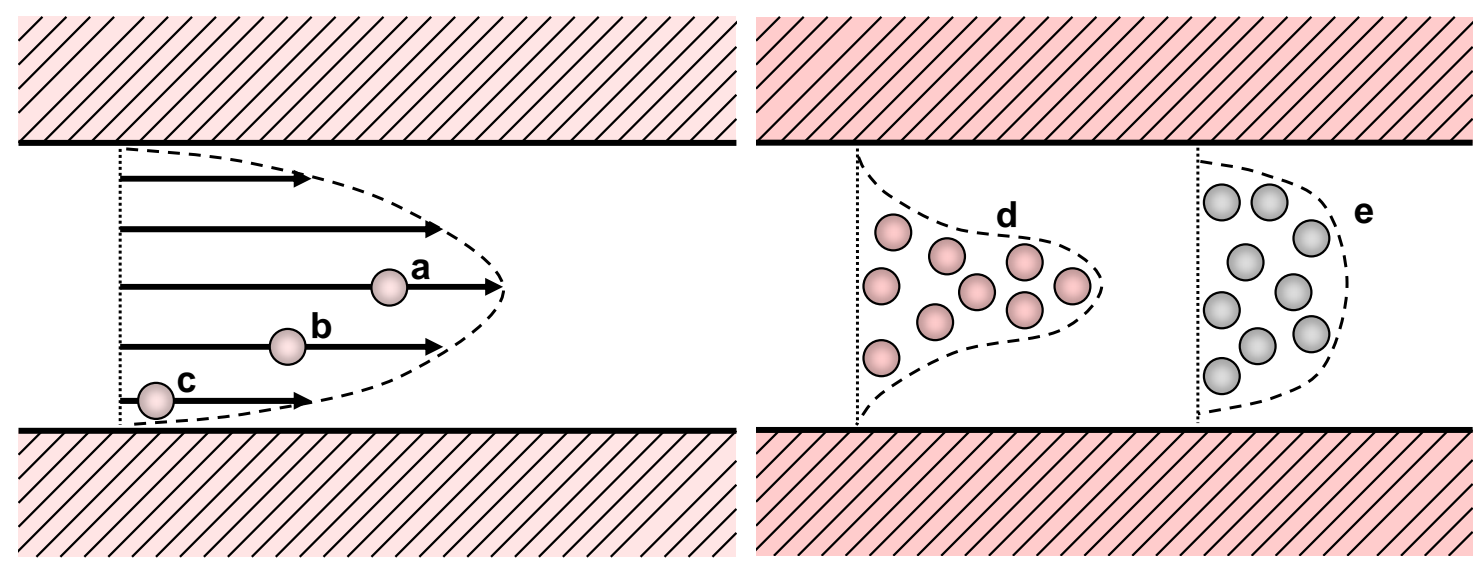

Fig. 3. Principle underlying faster migration of particles that are repelled by pore walls during flow of a suspension. Left: The expected parabolic flow velocity profile favors faster movement of particles near the center of flow (a) relative to near the pore walls (c). Right: Particles that are repelled from the walls tend to be enriched near the center of flow (d). Particles that are neither attracted nor repelled from the walls are still influenced by Brownian motion and a hydrodynamic effect that favors movement away from the pore wall (e).

Chun (2005) predicted a partitioning effect, with particles having higher attraction to pore walls eluting later in comparison to particles that are less attracted to the walls. Johnson et al. (2007) considered mechanical filtering effects to explain hindered elution of colloidal particles in the presence of repulsive colloidal forces between the surfaces.

Substantial delay in permeation can be expected in cases where colloidal particles are deposited onto the pore walls for at least part of the time that they are migrating within porous media (James and Chrysikopoulos 1999, 2000; Bauer and Nadler 2006; Simunek et al. 2006). Qian and Sen (2006) considered the case in which the particles spend part of the time in an attached state and derived predictive equations for net diffusion rates.

Although, as mentioned above, there are many reasons to expect that colloidal particles may become stuck or delayed in their transport through porous media, elution of particles through a packed bed can be substantially more rapid in comparison with smaller solute materials. As noted by McKay et al. (2000) and Zvikelsky and Weisbrod 2006), colloidal tracer particles can tend to elute as a "pulse," since they will tend to be

Wu et al. (2009). "Polyelectrolyte permeation in cellulose," BioResources 4(3), 1222-1262. 1246 
excluded from smaller pores and remain in relatively large channels. Such channels, such as the fractures in a mineral formation, can be expected to carry a disproportionately large proportion of the net flow. This mechanism is in many respects identical to that of gel permeation chromatography, as mentioned earlier (Potchka 1993).

\section{ENABLERS FOR DIFFUSION AND FLOW}

There are many situations in which one would like to speed up the penetration of solutes or colloidally-dispersed substances into cellulosic material. Such issues have occupied the attention of technologists in the wood, paper, and biofuels industries for many years. The goal of this section is to highlight relevant published work in this area, as well as to bring together some of the points already mentioned earlier in this article.

\section{Heating}

Raising the temperature can make sense both in terms of flow and in terms of solute diffusion rates. Fluid viscosities generally fall with increasing temperature. For example, if one raises the temperature of water from $25{ }^{\circ} \mathrm{C}$ to $60{ }^{\circ} \mathrm{C}$, the viscosity falls by a factor of two. The consequences can be judged, to a first approximation, by applying Eq. (3). Though the same trend does not necessarily hold true for a given solution of polyelectrolyte, raising the temperature is still a reasonable strategy to consider if one wants to increase flow rates at a given applied pressure.

Raising the temperature also can be expected to increase rates of diffusion. The fact that the viscosity coefficient appears in the denominator of Eq. (2), and also the absolute temperature appears in the numerator, means that the effect can be quite important.

A third way in which increased temperatures might increase permeation rates can be important in cases where the rate-limiting step in the process involves an energy of activation. For example, a polyelectrolyte or colloidal particle may have to pass through a zone of electrostatic repulsion in order to enter a pore. Such repulsion implies that the free energy of interaction is unfavorable, and diffusing entities will have to overcome a barrier of free energy to pass. The Arrhenius equation predicts, in analogous cases, that reaction rates tend to double with each 10-degree rise in temperature on the Celsius scale.

\section{Pressure}

Increased applied pressure is another almost self-evident strategy to increase permeation. As shown by Jeong and Corapcioglu (2005), increased pressure can be especially important if the viscous drag forces of the fluid are being used to displace blobs of a second phase, as in the case of soil remediation or enhanced oil recovery. However, sometimes excessive pressure can have the opposite effect by dislodging fine particulate matter that is present within porous media; such particles then may clog pores further downstream, often decreasing permeability to a drastic extent (Khilar and Fogler 1987; Hubbe et al. 2009). 


\section{Strategies to Widen Cellulosic Pores}

During the current push to develop cellulosic biofuels technology there has been considerable emphasis on finding ways to increase the accessibility of cellulosic biomass to enzymes, as well as to various reagents (see, for example, Kim and Lee 2002; Hu et al. 2008). Some strategies to accomplish this objective can be summarized by the terms delignification, refining, swelling, and enzymatic treatment.

\section{Delignification}

Removal of lignin is currently practiced as a highly mature, large-scale industry. The annual world production of kraft pulp is over 100 million tons (Anon. 2000). As shown by Stone and Scallan (1968), delignified fibers tend to be considerably more porous in comparison to mechanically separated wood fibers. Though chemical pulping often removes about half of the solid material from wood, the result can be quite favorable from the standpoint of producing ethanol and related products; in principle the cellulose can be hydrolysed to sugars, which then can be fermented with ordinary yeast into ethanol (Taherdazeh and Karimi 2007a,b). One of the slow steps in this process can be due to the limited accessibility of the cellulose matrix to cellulase enzymes. These protein structures may be $5-20 \mathrm{~nm}$ in size; this means in theory that the enzymes can enter many of the pores in cellulosic materials, especially after kraft pulping. Of course the degree to which enzymes actually permeate cellulose may depend on binding affinity and other factors.

\section{Refining}

In the manufacture of paper often the next key operation after pulp production involves passing a fiber slurry between rotating plates with raised rectangular bars, i.e. refining (Laine et al. 2004). During refining, groups of wetted fibers are repeatedly compressed and sheared. This mechanical treatment causes progressive delamination of the cell walls, and the outer layers of the fiber partially unravel, resulting in a fibrillated surface. As shown by Strazdins (1980), refining increases both the hydrodynamic surface area of the pulp, as well as the adsorption capacity of the pulp for cationic polymers. Conditions that favor greater swelling of fibers often can make refining somewhat more efficient, decreasing the amount of energy that is required to achieve a given level of fibrillation (Lindström and Kolman 1982).

\section{Swelling}

The subject of fiber swelling was already mentioned in the context of salt ions, which tend to shrink the pores within cellulosic fibers. If one wants to go the other direction and widen those pores, then one of the basic approaches involves raising the $\mathrm{pH}$. Cellulose fibers tend to have increased water-holding capacity with increased $\mathrm{pH}$, especially over the $\mathrm{pH}$ range between about 4 and 8 (Jayme and Büttel 1964). Such effects tend to become more important with increasing carboxyl group content of the fibers (Lindström and Carlsson 1982). 


\section{Enzymatic treatments}

Though enzymes may be hindered from entering the finest pores within the cell walls of fibers, fiber lumens are much larger. Thus, as was mentioned earlier, cellulase has been used to open up the bordered pit areas that connect the lumen space of one fiber with its nearest neighbors (Meyer 1974; Jacobs-Young et al. 1998). Analogously, in the field of enhanced oil recovery Lazar et al. (2007) introduced the use of enzymes to selectively break down some components of crude petroleum to improve its flow characteristics.

\section{Lowering of interfacial tension}

Surfactants can be used to accelerate penetration of certain other chemicals into cellulosic materials. Petrova et al. (1979) studied the effect of a nonionic surfactant on the impregnation of $\mathrm{NaOH}$ into birch and larch chips. The nonionic surfactant favored the penetration of alkali into the wood particles, as well as a partial dispersion and dissolution of wood components, resulting in loosening of wood structure and hence in better impregnation. Weignand et al. (2007) found that micro-emulsification of an amino-silicone greatly increased the efficiency of wood impregnation.

Brown and Jaffe (2001) found that certain nonionic surfactants facilitated the transport of bacterial cells through a sand filter. Though an explanation was offered, based on electrokinetic effects, it seems more likely that the effect was due to steric stabilization of the suspended matter (Fleer et al. 1993).

For cases involving displacement of oil from water-saturated porous media, various researchers have proposed that conditions for oil mobilization could be optimized based on the principles of interfacial tensions (Novozhilova et al. 1991; El-Batanoney et al. 1999; Abdel-Moghny 2004; Babadagli 2006). For instance, in a study aimed at removing residual oil from contaminated soil, Ang and Abdul (1991) found that the addition of surfactant had beneficial effects on displacement of the oil through the pores, dispersion of the oil into droplets due to the reduced interfacial tension, and solubilization of some of the oil in the water phase as micelles. In many of the cited studies favorable results have been obtained when the surfactants or pairs of surfactants were adjusted to obtain the lowest feasible interfacial tension. Summ and Soboleva (2003) found that injected emulsions were much more effective than simple surfactant solutions for the purpose of displacing dodecane from pores within a bed of quartz sand.

\section{Colloids and piggy-backing}

For purposes of enhancing the permeation of small but highly absorbing species, one strategy is to adsorb those materials onto colloidal particles. Researchers have identified this mechanism in connection with the movement of heavy metals and other contaminants in groundwater (Li et al. 2004; Simunek et al. 2006; Johnson et al. 2007). The approach was studied mathematically and in the lab by Grolimund and Borkovec (2005). It was found that results were influenced by adsorbed divalent ions, which can be expected to affect the colloidal stability of the particles. 
Anti-fouling polyelectrolyte layer

An innovative way to maintain the permeability of certain porous substrates in contact with potential contaminants can involve treatment with a water-loving polyelectrolyte. Thus Dainiak et al. (2002) employed a shielding layer of high-mass poly(acrylic acid) to minimize deposition of yeast cells on an ion exchange resin, while allowing binding of protein. A similar strategy might be used to minimize clogging of other porous systems.

\section{Electro-osmosis}

As noted by Park (1995), application of an electric field to a porous system that contains a diffusing polyelectrolyte will tend to orient the macromolecules in the field's direction. The convective velocity of the polyelectrolyte in a porous gel can be accelerated by this effect. Though the cited work was aimed at separation and analysis of DNA, it is possible that a similar approach could find applications in the treatment of cellulosic materials.

\section{Salt optimization / affinity optimization}

In the special case where the diffusing species is attracted to the pore walls, several studies have pointed to the need to optimize the aqueous conditions to favor permeation. On the one hand the attraction to the pore walls can provide a driving force for permeation to occur. But if the attraction is too strong, then, as was found by Horvath et al. (2008), the solute species may merely attach themselves near to the outer surfaces of the porous material and then remain fixed. Kluijtmans et al. (1998) found evidence that tracer particles may become trapped at pore junctions under condition of very low ionic strength, due to electrostatic forces. These effects were not observed at a higher salt level.

\section{CONCLUDING REMARKS}

Published sources cited in this article indicate that a number of different factors and mechanisms can affect permeation of simple ions, surfactants, polyelectrolytes, and colloids into the pores of cellulosic material immersed in water. It is evident that much progress has been made, using a broad range of experimental and theoretical approaches.

Despite the progress that has been made with respect to permeation into waterswollen wood and cellulosic fibers, one has to express some admiration for the somewhat recalcitrant nature of the material. Under ordinary conditions of pulping, refining, and swelling, the pores within the cell walls of cellulosic fibers still tend to remain small enough to discourage rapid enzymatic hydrolysis (Taherzadeh 2007b). Maybe this is a good thing. If cellulose were much more accessible, then one could expect much too rapid decay of standing trees and standing houses. We can expect that cellulosic materials will provide many challenges in the years ahead to technologists who choose to enter this field. 


\section{ACKNOWLEDGMENT}

Acknowledgment is made to the donors of the American Chemical Society Petroleum Research Fund for support of this research.

\section{LITERATURE CITED}

Abdel-Moghny, T. (2004). "Effect of mixed surfactant on the wettability and oil recovery of kaolin-oil-asphaltene system," J. Chinese Inst. Chem. Eng. 35(4), 455-465.

Alanissila, T., and Ying, S. C. (1992). "Theory of classical surface diffusion," Prog. Surface Sci. 39(3), 227-323.

Alince, B., and van de Ven, T. G. M. (1997). "Porosity of swollen pulp fibers evaluated by polymer adsorption," in C. F. Baker, ed., The Fundamentals of Papermaking Materials, Pira Intl., Leatherhead, Surrey, UK, 771-788.

Alvarez, A. C., Hime, G., Marchesin, D., and Bedrikovetsky, P. G. (2007). "The inverse problem of determining the filtration function and permeability reduction in flow of water with particles in porous media," Transport in Porous Media 70(1), 43-62.

Andersson, C. M., and Jarnström, L. (2006). "Controlled penetration of starch and hydrophobic sizing agent in surface sizing of porous materials," Appita J. 59(3), 207212.

Andrews, N. C., McHugh, A. J., and Schieber, J. D. (1998). "Polyelectrolytes in shear and extensional flows: Conformation and rheology," J. Polymer Sci. Part BPolymer Physics 36(8), 1401-1417.

Ang, C. C., and Abdul, A. S. (1991). "Aqueous surfactant washing or residual oil contamination from sandy soil," Ground Water Monitoring and Remediation 11(2), 121-127.

Anon. (2000). "P\&B breaks the 300 million ton barrier," Pulp Paper International 41(7), $10-11$.

Aspler, J. S., Davis, S., and Lyne, M. B. (1987). "The surface chemistry of paper in relation to dynamic wetting and sorption of water and lithographic fountain solution," J. Pulp Paper Sci. 13(2), J55-J60.

Auset, M., Keller, A. A., Brissaud, F., and Lazarova, V. (2005). "Intermittent filtration of bacteria and colloids in porous media," Water Resources Res. 41(9), article no. W09408.

Babadagli, T. (2006). "Evaluation of the critical parameters in oil recovery from fractured chalks by surfactant injection," J. Petroleum Sci. Eng. 54(1-2), 43-54.

Babadagli, T. (2007). "Development of mature oil fields - A review," J. Petroleum Sci. Eng. 57(3-4), 221-246.

Barrande, M., Bouchet, R., and Denoyel, R. (2007). "Tortuosity of porous particles," Anal. Chem. 79(23), 9115-9121.

Bauer, D., Goyeau, B., and Gobin, D. (2008). "Large particle transport in porous media: Effect of pore plugging on the macroscopic transport properties," J. Porous Media 11(4), 343-360. 
Bauer, W. R., and Nadler, W. (2006). "Molecular transport through channels and pores: Effects of in-channel interactions and blocking," Proc. Nat. Acad. Sci. USA 103(31), 11446-11451.

Beer, M., Schmidt, M., and Muthukumar, M. (1997). "The electrostatic expansion of linear polyelectrolytes: Effects of gegenions, co-ions, and hydrophobicity," Macromol. 30(26), 8375-8385.

Bendzalova, M., Pekarovicova, A., Kokta, B. V., and Chen, R. (1996). “Accessibility of swollen cellulosic fibers," Cellulose Chem. Technol. 30(1-2), 19-32.

Berthold, J., and Salmén, L. (1997). "Effects of mechanical and chemical treatments on the pore-size distribution in wood pulps examined by inverse size-exclusion chromatography," J. Pulp Paper Sci. 23(6), J245-J254.

Bishop, M. T., Langley, K. H., and Karasz, F. E. (1986). "Diffusion of a flexible polymer in a random porous material," Phys. Rev. Let. 57, 1741-1744.

Bousfield, D. W., and Karles, G. (2004). "Penetration into three-dimensional complex porous structures," J. Colloid Interface Sci. 270(2), 396-405.

Brand, F., Dautzenberg, H., Jaeger, W., and Hahn, M. (1997). "Polyelectrolytes with various charge densities: Synthesis and characterization of diallyldiemethylammonium chloride-acrylamide copolymers," Angew. Makromol. Chem. 248(4286), 41-71.

Bristow, J. A. (1967). "Liquid absorption into paper during short time intervals," Svensk Papperstidn. 70(19), 623-629.

Brown, D. G., and Jaffe, P. R. (2001). "Effects of nonionic surfactants on bacterial transport through porous media," Environ. Sci. Technol. 35(19), 3877-3883.

Brown, R. (1828). "A brief account of microscopical observations made in the months of June, July and August, 1827, on the particles contained in the pollen in plants: and on the general existence of active molecules in organic and inorganic bodies," Phil. Mag. 4, 161-173.

Bygrave, G. and Englezos, P. (1998). "Fiber charge from potentiometric titration of kraft pulp and Donnan equilibrium theory," Nordic Pulp Paper Res. J. 13(3), 1998.

Calderwood, G. F. N., and Mardles, E. W. J. (1955). "The rate of flow of liquids into capillaries under the action of surface forces," J. Textile Inst. 46(3), T161-T170.

Carrington, S., Odell, J., Fisher, L., Mitchell, J., and Hartley, L. (1996). "Polyelectrolyte behaviour of dilute xanthan solutions: Salt effects on extensional rheology," Polymer 37(13), 2871-2875.

Chaplain, V., and Mills, P. (1992). "Simulation of the migration of colloidal particles in porous media - Application to the transport of contaminants," Sci. Total Environ. $123,451-457$.

Chavez-Rojo, M. A., Juarez-Maldonado, R., and Medina-Noyola, M. (2008). "Diffusion of colloidal fluids in random porous media," Phys. Rev. E. 77(4), article no. 040401.

Chen, S. B. (1998). "Electrostatic interaction and hindered diffusion of ion-penetrable spheres in a slit pore," J. Colloid Interface Sci. 205(2), 354-364.

Choi, J. G., Do, D. D., and Do, H. D. (2001). "Surface diffusion of adsorbed molecules in porous media: Monolayer, multilayer, and capillary condensation regimes," Ind. Eng. Chem. Res. 40(19), 4005-4031. 
Chun, M. S. (2005). "Theoretical investigation regarding the hindered behavior of colloidal suspensions of nonuniformly charged nanoparticles in microfluidic devices," J. Indus. Eng. Chem. 11(3), 368-374.

Cooper, P. A. (1998). "Diffusion of copper in wood cell walls following vacuum treatment," Wood Fiber Sci. 30(4), 382-395.

Cule, D., and Hwa, T. (1998). "Polymer reptation in disordered media," Phys. Rev. Let. 80(14), 3145-3148.

Dainiak, M. B., Galaev, I. Y., and Mattiasson, B. (2002). "Polyelectrolyte-coated ion exchangers for cell-resistant expanded bed adsorption," Biotechnol. Prog. 18(4), 815820.

Davis, K. A., and Van Hise, J. R. (2006). "Comment on 'Predicting boron diffusion in wood from surface sorption'," Forest Products J. 56(6), 38-39.

De, D., Hrymak, A. N., and Pelton, R. (1997). "Polyelectrolyte precipitate formation during miscible displacement in porous media," AIChE J. 43(10), 2415-2423.

Dechadilok, P., and Deen, W. M. (2006). "Hindrance factors for diffusion and convection in pores," Indust. Eng. Chem. Res. 45(21), 6953-6959.

de Gennes, P. G. (1971). "Reptation of a polymer chain in presence of fixed obstacles," J. Chem. Phys. 55, 572-579.

Delgado, J. M. P. Q. (2006). "A critical review of dispersion in packed beds," Heat Mass Trans. 42(4), 279-310.

Dimitrov, D. I., Milchev, A., and Binder, K. (2007). "Capillary rise in nanopores: Molecular dynamics evidence for the Lucas-Washburn equation," Phys. Rev. Let. 99(5), article 054501.

Dourado, F., Gama, F. M., Chibowski, E., and Mota, M. (1998). "Characterization of cellulose surface free energy," J. Adhesion Sci. Technol. 12(10), 1081-1090.

Dudko, O. K., Berezhkovskii, A. M., and Weiss, G. H. (2005). "Time-dependent diffusion coefficients in periodic porous materials," J. Phys. Chem. B 109(45), 21296-21299.

Durr, M., Schmid, A., Obermaier, M., Yasuda, A., and Nelles, G. (2005). "Diffusion properties of dye molecules in nanoporous $\mathrm{TiO}_{2}$ networks," J. Phys. Chem. A 109(17), 3967-3970.

Einstein, A. (1905). "Concerning the motion of particles suspended in quiescent liquids based on the molecular kinetic theory of heat," Ann. Phys. 17, 549-560.

Ek, R., Henriksson, U., Nystrom, C., and Odberg, L. (1994). "Pore characterization in cellulose beads from diffusion studies using the spin-echo NMR technique," Powder Technol. 81(3), 279-286.

Eklund, D., and Salminen, P. (1987). "Water sorption in paper during short times," Appita 40(5), 340-346.

El-Batanoney, M., Abdel-Moghny, T., and Ramzi, M. (1999). "The effect of mixed surfactants on enhancing oil recovery," J. Surfactants Detergents 2(2), 201-205.

Ershov, A. P., Zorin, Z. M., Sobolev, V. D., and Churaev, N. V. (2003). "Capillary flow of cationic polyelectrolyte solutions," Colloid J. 65(4), 434-439.

Everett, D. H., Haynes, J. M., and Miller, R. J. L. (1977). "Kinetics of capillary imbibition by fibrous materials," in: Fiber-Water Interactions in Paper-making, The British Paper and Board Industry Federation, London, 519-536.

Wu et al. (2009). "Polyelectrolyte permeation in cellulose," BioResources 4(3), 1222-1262. 1253 
Fält, S., and Wågberg, L. (2003). "Influence of electrolytes on the swelling and strength of kraft-liner pulps," Nordic Pulp Paper Res. J. 18(1), 69-73.

Farley, C. E. (1997). "Factors influencing the rate of charge decay," Tappi J. 80(10), 177183.

Fick, A. (1855). "On liquid diffusion," Phil. Mag. 10, 30-39.

Fisher, I., and Eizenberg, M. (2008). "Copper ion diffusion in porous and nonporous SiO2-based dielectrics using bias thermal stress and thermal stress tests," Thin Solid Films 516(12), 4111-4121.

Fleer, G. J., Cohen Stuart, M. A., Scheutjens, J. M. H. M., Cosgrove, T., and Vincent, B. (1993). Polymers at Interfaces, Chapman and Hall, London.

Gauthier, M. G., and Slater, G. W. (2008). "A Monte Carlo algorithm to study polymer translocation through nanopores. I. Theory and numerical approach," J. Chem. Phys. 128(6), article no. 065103.

Grolimund, D., and Borkovec, M. (2005). "Colloid-facilitated transport of strongly sorbing contaminants in natural porous media: Mathematical modeling and laboratory column experiments," Environ. Sci. Technol. 39(17), 6378-6386.

Gruber, E., Grossmann, K., and Schempp, W. (1996). "Interactions of synthetic cationic polymers with fibers and fillers; influence on adsorption," Wochenbl. Papierfabr. 124(1), 4-11.

Grznarova, G., Yu, S., Stefuca, V., and Polakovic, M. (2005). “Quantitative characterization of pore structure of cellulose gels with or without bound protein ligand," J. Chromatog. 1092(1), 107-113.

Guo, Y. H., Langley, K. H., and Karasz, F. E. (1990). "Time scale dependence of diffusion in porous material - Dynamic light scattering and computer simulation," $J$. Chem. Phys. 93, 7457-7462.

Gustafson R. R., Jiménez, G., McKean, W. T., and Chian, D.-S. (1997). "The role of penetration in nonuniform pulping of softwood chips," Tappi J. 72(8), 163-167.

Gutsche, R., and Yoshida, H. (1994). "Solid diffusion in the pores of cellulose membrane," Chem. Eng. Sci. 49(2), 179-188.

Herrington, T. M., and Petzold, J. C. (1992). "An investigation into the nature of charge on the surface of papermaking woodpulps. 2. Analysis of Potentiometric data," Colloids Surf. 64, 109-118.

Hiemenz, P. C., and Lodge, T. P. (2007). Polymer Chemistry, CRC Press, Boca Raton.

Hiemenz, P. C., and Rajagopalan, R. (1997). Principles of Colloid and Surface Science, $3^{\text {rd }}$ Ed., Dekker, New York.

Ho, Y. S., Ng, J. C. Y., and McKay, G. (2000). "Kinetics of pollutant sorption by biosorbents: Review," Separation Purification Methods 29(2), 189-232.

Hostetler, R. E., and Swanson, J. W. (1974). "Diffusion into and adsorption of polyethylenimine on porous silica gel," J. Polymer Sci. 12(1), 29-43.

Horvath, A. T., Horvath, A. E., Lindström, T., and Wågberg, L. (2008). "Diffusion of cationic polyelectrolytes into cellulosic fibers," Langmuir 24(19), 10797-10806.

Hu, G., Heitmann, J. A., and Rojas, O. J. (2008). "Feedstock pretreatment strategies for producing ethanol from wood, bark, and forest residues," BioRes. (3(1), 270-294.

Hubbe, M. A. (2006). "Sensing the electrokinetic potential of cellulosic fiber surfaces," BioRes. 1(1), 116-149. 
Hubbe, M. A. (2007). "Paper's resistance to wetting - A review of internal sizing chemicals and their effects," BioRes. 2(1), 106-145.

Hubbe, M. A., Rojas, O. J., Lee, S. Y., Park, S., and Wang, Y. (2007a). "Distinctive electrokinetic behavior of nanoporous silica particles treated with cationic polyelectrolyte," Colloids Surf. A. 292(2-3), 271-278.

Hubbe, M. A., Rojas, O. J., Lucia, L. A., and Jung, T. M. (2007b). "Consequences of the Nanoporosity of Cellulosic Fibers on their Streaming Potential and their Interactions with Cationic Polyelectrolytes," Cellulose 14(6), 655-671.

Hubbe, M. A., Chen, H., and Heitmann, J. A. (2009). "Permeability reduction phenomena in packed beds, fiber mats, and wet webs of paper exposed to flow of liquids and suspensions: A review," BioRes. 4(1), 405-451.

Hunt, A. G. (2005). "Continuum percolation theory for transport properties in porous media," Philosophical Mag. 85(29), 3409-3434.

Idol, W. K., and Anderson, J. L. (1986). "Effects of adsorbed polyelectrolytes on convective flow and diffusion in porous membranes," J. Membrane Sci. 28(3), 269286.

Inglezakis, V. J., Diamandis, N. A., Loizidou, M. D., and Grigoropoulou, H. P. (1999). "Effect of pore clogging on kinetics of lead uptake by clinoptilolite," J. Colloid Interface Sci. 215(1), 54-57.

Ito, R., Golman, B., and Shinohara, K. (2003). "Effect of the porous structure of a coating layer on the release rate of core particle," J. Chem. Eng. Japan 36(6), 676-682.

Iveson, S. M., Rutherford, K. F., and Biggs, S. R. (2001). "Liquid penetration rate into submerged porous particles: theory, experimental validation and implications for iron ore granulation and sintering," Trans. Inst. Mining Metallurgy Sec. C-Mineral Proc. Extrac. Metallury 110, C133-C143.

Jacobson, A. J., and Banerjee, S. (2006). "Diffusion of tritiated water into water-saturated wood particles," Holzforschung 60(1), 59-63.

Jacobs-Young, C. J., Venditti, R. A., and Joyce, T. W. (1998). "Effect of enzymatic pretreatment on the diffusion of sodium hydroxide in wood," Tappi J. 81(1), 260-266.

James, S. C., and Chrysikopoulos, C. V. (1999). "Transport of polydisperse colloid suspensions in a single fracture," Water Resources Res. 35(3), 707-718.

James, S. C., and Chrysikopoulos, C. V. (2000). "Transport of polydisperse colloids in a saturated fracture with spatially variable aperture," Water Resources Res. 36(6), 1457-1465.

Jayme, G., and Büttel, H. (1964). "The dependency of water retention value (WRV) and strength properties on the $\mathrm{pH}$ value during refining for various bleached and unbleached cellulose pulps," Wochenbl. Papierfabr. 92(23/24), 718-727.

Jeong, S. W., and Corapcioglu, M. Y. (2005). "Force analysis and visualization of NAPL removal during surfactant-related floods in a porous medium," J. Hazaardous Materials 126(1-3), 8-13.

Jeremic, D., Cooper, P., and Brodersen, P. (2007). "Penetration of poly(ethylene glycol) into wood cell walls of red pine," Holzforschung 61(3), 272-278.

Jervis, E. J., Haynes, C. A., and Kilburn, D. G. (1997). "Surface diffusion of cellulases and their isolated binding domains on cellulose," J. Biological Chem. 272(38), 24016-24023. 
Johnson, W. P., Tong, M., and Li, X. (2007). "On colloid retention in saturated porous media in the presence of energy barriers: The failure of alpha, and opportunities to predict eta," Water Resources Res. 43(12), article no. W12S13.

Kabanov, V. A., Zezin, A. B., and Rogacheva, V. B. (1989). "Active transport of linear polyions in oppositely charged swollen polyelectrolyte networks," Macromol. Chem. Phys. 190, 2211-2216.

Kamke, F. A., and Lee, J. N. (2007). “Adhesive penetration in wood - A review," Wood Fiber Sci. 39(2), 205-220.

Kanungo, M., and Collinson, M. M. (2003). "Diffusion of redox probes in hydrated solgel-derived glasses. Effect of gel structure," Anal. Chem. 75(23), 6555-6559.

Khilar, K. C., and Fogler, H. S. (1987). "Colloidally induced fines migration in porous media," Rev. in Chem. Eng. 4(1-2), 41-108.

Kim, H., Cho, C. H., and Lee, E. K. (2005). "Dynamics of simple fluids confined in cylindrical pore: Effect of pore size," J. Theor. Comput. Chem. 4(1), 305-315.

Kim, J. T.., and Anderson, J. L. (1991). "Diffusion and flow through polymer-lined micropores," Indust. Eng. Chem. Res. 30(5), 1008-1016.

Kim, S. B., and Lee, Y. Y. (2002). "Diffusion of sulfuric acid within lignocellulosic biomass particles and its impact on dilute-acid pretreatment," BioRes. Technol. 83(2), 165-171.

Kjaergaard, C., Poulsen, T. G., Moldrup, P., and de Jonge, L. W. (2004a). “Colloid mobilization and transport in undisturbed soil columns. I. Pore structure characterization and tritium transport," Vadose Zone J. 3(2), 413-423.

Kluijtmans, S. G. J. M., de Hoog, E. H. A., and Philipse, A. P. (1998). "Self-diffusion of charged colloidal tracer spheres in transparent porous glass media: Effect of ionic strength and pore size," J. Chem. Phys. 108(17), 7469-7477.

Koethe, J. L., and Scott, W. E. (1993). "Polyelectrolyte interactions with papermaking fibers: the mechanism of surface-charge decay," Tappi J. 76(12), 123-133.

Kosakowski, G. (2004). "Anomalous transport of colloids and solutes in a shear zone," $J$. Contaminant Hydrology 72(1-4), 23-46.

Kumar, R., and Muthukumar, M. (2008). "Confinement free energy of flexible polyelectrolytes in spherical cavities,” J. Chem. Phys. 128(18), article 184902.

Kunetz, J., and Hench, L. (1998). "Restricted diffusion of chromium nitrate salt solutions into porous sol-gel-silica monoliths," J. Amer. Ceramic Soc. 81(4), 877-884.

Lai, S. K., Kau, C. Y., Tang, Y. W., and Chan, K. Y. (2004). "Anomalous diffusivity and electric conductivity for low concentration electrolytes in nanopores," Phys. Rev. E 69(5), article no. 051203.

Laine, C., Wang, X. S., Tenkanen, M., and Varhimo, A. (2004). "Changes in the fiber wall during refining of bleached pine kraft pulp," Holzforschung 58(3), 233-240.

Lazar, I., Petrisor, I. G., and Yen, T. E. (2007). "Microbial enhanced oil recovery (MEOR)," Petroleum Sci. Technol. 25(11-12), 1353-1366.

Lebon, G., Desaive, T., and Dauby, P. (2006). "A unified extended thermodynamic description of diffusion, thermo-diffusion, suspensions, and porous media," J. Appl. Mechanics - Trans. ASME 73(1), 16-20.

Levenspiel, O., and Smith, W. K. (1957). "Notes on the diffusion-type model for the longitudinal mixing of fluids in flow," Chem. Eng. Sci. 6(4-5), 227-233.

Wu et al. (2009). "Polyelectrolyte permeation in cellulose," BioResources 4(3), 1222-1262. 1256 
Levenspiel, D. (1958). "Longitudinal mixing of fluids flowing in circular pipes," Ind. Eng. Chem. 50(3), 343-346.

Leyvaramos, R., and Geankoplis, C. J. (1994). "Diffusion in liquid-filled pores of activated carbon. 1. Pore volume diffusion," Can. J. Chem. Eng. 72(2), 262-271.

Li, S., and Dai, H. (2004). "The influences of inorganic salts on WRV of papermaking stock," Proc. $2^{\text {nd }}$ Internat. Symp. on Technol. Pulping, Papermaking, Biotechnol. Fiber Plants $\left(2^{\text {nd }} I S T P P B F P\right)$, Nanjing, China, Oct. 13-14, 2004, Nanjing Forestry Univ., 434-473.

Li, S. H., Yang, H. T., and Jen, C. P. (2004). "Modeling of colloid transport mechanisms facilitating migration of radionuclides in fractured media," Nuclear Technol. 148(3), 358-368.

Li, T. Q., Henriksson, J., Klason, T., and Odberg, L. (1992). "Water diffusion in wood pulp cellulose fibers studied by means of the pulsed gradient spin-echo method," $J$. Colloid Interface Sci. 154(2), 305-315.

Lin, K.-F., and Cheng, H.-L. (2000). "A simple method to estimate chain conformations of polyelectrolytes in the semidilute regime," Macromol. 33, 4961-4965.

Lin, L., and Jorge, H. R. (2005). "Analysis and modelling of wicking through carton liquid packaging," Anal. Chim. Acta 552(1-2), 218-225.

Linden, T., Ljunglof, A., Kula, M.-R., and Thommes, J. (1999). "Visualizing twocomponent protein diffusion in porous adsorbents by confocal scanning laser microscopy," Biotechnol. Bioeng. 65, 622-630.

Lindström, T., and Carlsson, G. (1982). "The effect of carboxyl groups and their ionic form during drying on the hornification of cellulosic fibers," Svensk Papperstidn. 85(15), R146-R151.

Lindström, T., and Kolman, M. (1982). "Effect of pH and electrolyte concentration during beating and sheet forming on paper strength," Svesnk Papperstidn. 85( ), R143-R145.

Linke, G. T., Lipowsky, R., and Gruhn, T. (2006). "Osmotically induced passage of vesicles through narrow pores," Europhys. Let. 74(5), 916-922.

Lönnberg, B., Robertson, L., and Saari, K. (1992). "Chemical diffusion in wood," in AIChE Forest Products Symposium.

Lua, R. C., and Grosberg, A. Y. (2005). "First passage times and asymmetry of DNA translocation," Phys. Rev. E 72(6), article no. 061918.

Lyne, M. B., and Aspler, J. S. (1982). "Wetting and sorption of water by paper under dynamic conditions," Tappi J. 65(12), 98-101.

Lyne, M. B., and Huang, Y. C. (1993). "Measuring acid-base and dispersive interactions with paper surfaces under dynamic conditions," Nordic Pulp Paper Res. J. 8(1), 120122.

Maattanen, M., and Tikka, P. (2008). "Penetration of water and black liquor into overthick and treated wood chips," Paperi Puu 90(2), 34-39.

Maekawa, M., Murkami, K., and Yoshida, H. (1995). "Effects of type of adsorption isotherms on parallel diffusion of sulfonated dyes into porous cellulose membrane," Colloid Polymer Sci. 273(8), 793-799. 
Malkov, S., Tikka, P., and Gullichsen, J. (2003). "Towards complete impregnation of wood chips with aqueous solutions. Part I. A retrospective and critical evaluation of the penetration process," Paperi Puu 85(8), 460-466.

Maloney, T. C., Paulapuro, H., and Stenius, P. (1998). "Hydration and swelling of pulp fibers measured with differential scanning calorimetry," Nordic Pulp Paper Res. J. 13(1), 31-36.

Mays, D. C., and Hunt, J. R. (2005). "Hydrodynamic aspects of particle clogging in porous media," Environ. Sci. Technol. 39(2), 577-584.

McKay, L. D., Sanford, W. E., and Strong, J. M. (2000). "Field-scale migration of colloidal tracers in a fractured shale saprolite," Ground Water 38(1), 139-147.

Meyer, R. W. (1974). "Effect of enzyme treatment on bordered-pit ultrastructure, permeability, and toughness of the sapwood of three western conifers," Wood Sci. 6(3), 220-230.

Miklavic, S. J. (1993). "Self-consistent field theory for confined polyelectrolyte chains," J. Phys. Chem. 97, 3924-3926.

Miller, M. D., and Bruening, M. L. (2005). "Correlation of the swelling and permeability of polyelectrolyte multilayer films," Chem. Mater. 17(21), 5375-5381.

Mitra, S., Mukhopadhyay, R., Tsukushi, I., and Ikeda, S. (2001). "Dynamics of water in confined space (porous alumina): QENS,” J. Phys. - Condensed Matter 13(37), 84558465.

Muralidhar, R., and Ramkrishna, D. (1993). "Diffusion in pore fractals - A review of linear-response models," Transport in Porous Media 13(1), 79-95.

Nakashima, Y. (2003). "Diffusion of $\mathrm{H}_{2} \mathrm{O}$ in smectite gels: Obstruction effects of bound $\mathrm{H}_{2} \mathrm{O}$ layers," Clays Clay Mater. 51(1), 9-22.

Nelissen, K., Misko, V. R., and Peeters, F. M. (2007). "Single-file diffusion of interacting particles in a one-dimensional channel," EPL 80(5), article no. 56004.

Neuman, R. P., and Walker, L. P. (1992). "Solute exclusion from cellulose in packed columns - Process modeling and analysis," Biotechnol. Bioeng. 40(2), 226-234.

Nguyen, T. Q., and Kausch, H.-H. (1992). "Chain extension and degradation in convergent flow," Polymer 33(12), 2611-2621.

Nitsche, J. M., and Balgi, G. (1994). "Hindered Brownian diffusion of spherical solutes witin circular cylindrical pores," Indust. Eng. Chem. Res. 33(9), 2242-2247.

Novozhilova, O. G., Mekhtiev, F. D., Kirsanov, E. A., Matveenko, V. N., and Schukin, E. D. (1991). "Colloidal chemical aspects of displacement of hydrocarbons from porous media," Colloid J. USSR 53(3), 475-478.

Obi, E. O., and Blunt, M. J. (2004). "Streamline-based simulation of advective-dispersive solute transport," Adv. Water Resources 27(9), 913-924.

Odell, J. A., and Haward, S. J. (2008). "Viscosity enhancement in the flow of hydrolysed poly(acrylamide) saline solutions around spheres: implications for enhanced oil recovery," Rheologica Acta 47(2), 129-137.

Olson, M. S., Ford, R. M., Smith, J. A., and Fernandez, E. J. (2005). “Analysis of column tortuosity for $\mathrm{MnCl}_{2}$ and bacterial diffusion using magnetic resonance imaging," Environ. Sci. Technol. 39(1), 149-154. 
Osaki, S., Inoue, E., Sugihara, S., and Takashima, Y. (1993). "Direct measurement of diffusion coefficients for $\mathrm{Sr}$ and $\mathrm{Co}$ in silica gel layers under various conditions," $J$. Radioanal. Nuclear Chem. 170(2), 381-387.

Oswald, J. G., and Ibaraki, M. (2001). "Migration of colloids in discretely fractured porous media: effect of colloidal matrix diffusion," J. Contaminant Hydrol. 52(1-4), 213-244.

Paranyi, N. I., and Rabinovitch, W. (1958). “ ," Pulp Paper Mag. Can. 58(10), 223 -

Park, Y. (1995). "The separation of polyelectrolyte using an electric field in a packed column," Separation Sci. Technol. 30(19), 3629-3647.

Pawar, Y., and Anderson, J. L. (1993). "Hindered diffusion in slit pores - An analytical result," Indust. Eng. Chem. Res. 32(4), 743-746.

Petel'skii, M. B., and Abramzon, A. A. (1999). "Specific features of the diffusion of surfactants through porous membranes," Russian J. Phys. Chem. 73(6), 964-967.

Petlicki, J., and van de Ven, T. G. M. (1994). "Adsorption of polyethylenimine onto cellulose fibers," Colloids Surf. A 83(1), 9-23.

Petrova, S. I., Senicheva, L. V., Maiorova, L. P, and Khrustaleva, L. G. (1979). "Effect of surfactants on low-temperature impregnation of wood with alkaline solutions," Bumazhnaia Promyshlennost (5), 20-21.

Potchka, M. (1993). "Mechanism of size-exclusion chromatography. 1. Role of convection and obstructed diffusion in size-exclusion chromatography," $J$. Chromatography 648(1), 41-69.

Qian, J., and Sen, P. N. (2006). "Time dependent diffusion in a disordered medium with partially absorbing walls: A perturbative approach," J. Chem. Phys. 125(19), article no. 194508.

Ra, J. B., Barnes, H. M., and Conners, T. E. (2001). "Determination of boron diffusion coefficients in wood," Wood Fiber Sci. 33(1), 90-103.

Ra, J. B., Barnes, H. M., and Conners, T. E. (2002). "Predicting boron diffusion in wood from surface sorption," Forest Products J. 52(10), 67-70.

Reeder, D. H., Carr, P. W., Flickinger, M. C., and McCormick, A. V. (2000). "Diffusion of nonadsorbing polymers within hierarchically structured colloidal aggregates," $J$. Colloid Interface Sci. 226(2), 277-285.

Revil, A. (1999). "Ionic diffusivity, electrical conductivity, membrane and thermoelectric potentials in colloids and granular porous media: A unified model," J. Colloid Interface Sci. 212(2), 503-522.

Robertson, L., and Lönnberg, B. (1991a). "Diffusion in wood. 1. Theory and apparatus," Paperi Puu 73(6), 532-535.

Robertson, L., and Lönnberg, B. (1991b). "Diffusion in wood. 2. The effects of concentration and temperature," Paperi Puu 73(7), 635, 637-639.

Saffman, P. G. (1965). "The lift on a small sphere in a slow shear flow," J. Fluid Mech. 22(2), 385-400.

Sahimi, M. (1993). "Fractal and superdiffusive transport and hydrodynamic dispersion in heterogeneous porous media," Transport in Porous Media 13(1), 3-40.

Sarmadi, A. M., Kwon, Y. A., and Young, R. A. (1993). "Wettability of nonwoven fabrics. 1. Effect of fluorochemical finishes on water repellency," Ind. Eng. Chem. Res. 32(2), 279-287. 
Saugey, A., Joly, L., Ybert, C., Barrat, J. L., and Bocquet, L. (2005). "Diffusion in pores and its dependence on boundary conditions," J. Phys. - Condensed Matter 17(49), S4075-S4090.

Scallan, A. M. (1983). "The effect of acidic groups on the swelling of pulps: A review," Tappi J. 66(11), 73-75.

Schultz, E. B., Matney, T. G., and Koger, J. L. (1999). "A neural network model for wood chip thickness distributions," Wood Fiber Sci. 31(1), 2-14.

Sekine, T., and Nakatani, K. (2002). "Intraparticle diffusion and adsorption isotherm for sorption in silica gel studied by single-microparticle injection and microabsorption methods," Langmuir 18(3), 694-697.

Sekine, T., and Nakatani, K. (2004). "Nanometer pore size dependence of intraparticle diffusion in silica gel," Chemistry Let. 33(5), 600-601.

Senden, T. J., Knackstedt, M. A., and Lyne, M. B. (2000). “Droplet penetration into porous networks: Role of pore morphology," Nordic Pulp Paper Res. J. 15(5), 554563.

Sernek, M., Resnik, J., and Kamke, F. A. (1999). "Penetration of liquid ureaformaldehyde adhesive into beech wood," Wood Fiber Sci. 31(1), 41-48.

Shaqfeh, E. S. G., and Koch, D. L. (1992). "Polymer stretch in dilute fixed beds of fibers or spheres," J. Fluid Mechanics 244, 17-54.

Shen, C. Y., Huang, Y. F., Li, B. G., and Jin, Y. (2008). "Effects of solution chemistry on straining of colloids in porous media under unfavorable conditions," Water Resources Res. 44(5), article no. W05419.

Shin, C. (2006). "Numerical simulation for particle penetration depth distribution in deep bed filtration," Cehm. Eng. Technol. 29(8), 905-909.

Siau, J. F. (1984). Transport Processes in Wood, Springer Ser. Wood Sci. Technol., Timmell, T. E. (ser. ed.), Syracuse, 225.

Simunek, J., He, C. M., Pang, L. P., and Bradford, S. A. (2006). "Colloid-facilitated solute transport in variably saturated porous media: Numerical model and experimental verification," Vadose Zone J. 5(3), 1035-1047.

Skaar, C., and Siau, J. F. (1981). "Thermal diffusion of bound water in wood," Wood Sci. Technol. 15, 105-112.

Srivastava, V. C., Mall, I. D., and Mishra I. M. (2006), “Characterization of mesoporous rice husk ash (RHA) and adsorption kinetics of metal ions from aqueous solution onto RHA," J. Hazardous Materials 134(1-3), 257-267.

Stamm, A. J. (1953). "Diffusion and penetration mechanism of liquids into wood," Pulp Paper Mag. Can. 54(2), 54-63.

Stamm, A. J. (1959). "Bound-water diffusion into wood in the fiber direction," Forest Prod. J. 9, 27-32.

Standnes, D. C. (2004). "Analysis of oil recovery rates for spontaneous imbibition of aqueous surfactant solutions into preferential oil-wet carbonates by estimation of capillary diffusivity coefficients," Colloids Surf. A 251(1-3), 93-101.

Starov, V. M. (2004). "Surfactant solutions and porous substrates: spreading and imbibition," Adv. Colloid Interface Sci. 111(1-2), 3-27. 
Starov, V. M., Zhdanov, S. A., and Velarde, M. G. (2004). "Capillary imbibition of surfactant solutions in porous media and thin capillaries: Partial wetting case," $J$. Colloid Interface Sci. 273(2), 589-595.

Strazdins, E. (1980). "New chemical approaches towards optimizing dry strength of stock furnishes containing waste paper," Das Papier 34(10A), V49V-V55.

Sud, D., Mahajan, G., and Kaur, M. P. (2008). "Agricultural waste material as potential adsorbent for sequestering heavy metal ions from aqueous solutions - A review," Biores. Technol. 99(14), 6017-6027.

Summ, B. D., and Soboleva, O. A. (2003). "Displacement of organic liquids from capillaries and porous media by aqueous surfactant solutions and organosiloxane-inwater emulsions," Theor. Found. Chem. Eng. 37(1), 8-13.

Stone, J. E., and Green, H. V. (1958). "Penetration and diffusion into hardwoods," Pulp Paper Mag. Can. 58(10), 223-232.

Stone, J. E., and Scallan, A. M. (1966). "Influence of drying on the pore structures of the cell wall," In: Bolam, F. (ed.), Consolidation of the Paper Web, Trans. Symp. Cambridge, Vol. 1, 145-174.

Stone, J. E., and Scallan, A. M., "A Structural Model of the Cell Wall of Water-Swollen Wood Pulp Fibers based on their Accessibility to Macromolecules," Cellulose Chem. Technol. 2 (3): 321-342 (1968).

Summ, B. D., and Soboleva, O. A. (2003). "Displacement of organic liquids from capillaries and porous media by aqueous surfactant solutions and organosiloxane-inwater emulsions," Theor. Found. Chem. Eng. 37(1), 8-13.

Swanson, J. W., and Cordingly, S. (1959). "Surface chemical studies of pitch. II. The mechanism of the loss of absorbency and development of self-sizing in papers made from wood pulps," Tappi 42(10), 812-819.

Taherdazeh, M. J., and Karimi, K. (2007a). "Acid-based hydrolysis processes for ethanol from lignocellulosic materials: A review," BioRes. 2(3), 472-499.

Taherzadeh, M. J., and Karimi, K. (2007b). "Enzyme-based hydrolysis processes for ethanol from lignocellulosic materials: A review," BioRes. 2(4), 707-738.

Tantemsapya, N., and Meegoda, J. N. (2004). "Estimation of diffusion coefficient of chromium in colloidal silica using digital photography," Environ. Sci. Technol. 38(14), 3950-3957.

Tatsumi, D., and Yamauchi, T. (1997). "Depth profile of paper additives within a pulp fiber," in The Fundamentals of Papermaking Materials, C. F. Baker, ed., Pira International, Leatherhead, Surrey, UK, Vol. 2, 789-814.

ten Bosch, A. (2001). "Persistent diffusion in nanopores," J. Chem. Phys. 114(11), 49824988.

Teraoka, I., Landley, K. H., and Karasz, F. E. (1992). "Reptation dynamics of semirigid polymers in porous media," Macromol. 25(23), 6106-6112.

Tollenaar (1954). "The rise of liquids into a wedge-shaped crevice," Appl. Sci. Res. Sec. A-Mechanics Heat Chem. Eng. Math. Methods 4(5-6), 453-456.

Topgaard, D., and Soderman, O. (2001). "Diffusion of water absorbed in cellulose fibers studied with H-1-NMR,” Langmuir 17(9), 2694-2702.

Törnqvist, M., Hurme, T., and Rosenholm, J. B. (2001). "Drift speed: A way of measuring diffusion and tortuosity of porous materials," Colloids Surf. A 180, 23-31.

Wu et al. (2009). "Polyelectrolyte permeation in cellulose," BioResources 4(3), 1222-1262. 1261 
Törnqvist, M., Hurme, T., and Rosenholm, J. B. (2004). "The effect of HS' ions in 1 $\mathrm{ml} / \mathrm{dm}^{3} \mathrm{NaOH}$ solutions on the ion diffusion in wood calculated by combining drift speed and steady state diffusion," Colloids Surf. A 232, 191-197.

Tovbin, Y. K. (2005). "Self-diffusion coefficients for pure and mixed adsorbate fluids in narrow pores," Adsorption - J. Intl. Adsorp. Soc. 11(3-4), 245-257.

Vargas-Florencia, D., Edvinsson, T., Hagfeldt, A., and Furo, I. (2007). "Pores in nanostructured $\mathrm{TiO}_{2}$ films. Size distribution and pore permeability," J. Phys. Chem. C 111(21), 7605-7611.

Veith, S. R., Hughes, E., Vuataz, G., and Pratsinis, S. E. (2004). "Restricted diffusion in silica particles measured by pulsed field gradient NMR," J. Colloid Interface Sci. 274(1), 216-228.

Wågberg, L. (2000). "Polyelectrolyte adsorption onto cellulose fibers - A review," Nordic Pulp Paper Res. J. 15(5), 586-597.

Wågberg, L., Ödberg, L., and Glad-Nordmark, G. (1989). "Charge determination of porous substrates by polyelectrolyte adsorption. Part 1. Carboxymethylated, bleached cellulosic fibers," Nordic Pulp Paper Res. J. 4(2), 71-76.

Wallstrom, L., and Lindberg, K. A. H. (1999). "Measurement of cell wall penetration in wood of water-based chemicals using SEM/EDS and STEM/EDS technique," Wood Fiber Sci. 33(2), 111-122.

Wang, C., and Luo, M. B. (2007). "Effect of interchain interactions on the translocation of polymer chains through small holes," J. Appl. Polymer Sci. 103(2), 1200-1205.

Wang, F., and Hubbe, M. A. (2002). "Charge properties of fibers in the paper mill environment. 1. Effect of electrical conductivity," J. Pulp Paper Science 28(10), 347353.

Wang, Y., Angelatos, A. S., Dunstan, D. E., and Caruso, F. (2007). "Infiltration of macromolecules into nanoporous silica particles," Macromol. 40(21), 7594-7600.

Wei, Q. H., Bechinger, C., and Leiderer, P. (2000). "Single-file diffusion of colloids in one-dimensional channels," Science 287(5453), 625-627.

Weigenand, O., Militz, H., Tingaut, P., Sebe, G., de Jeso, B., and Mai, C. (2007). "Penetration of amino-silicone micro- and macro-emulsions into Scots pine sapwood and the effect on water-related properties," Holzforschung 61(1), 51-59.

Weise, U., Maloney, T, and Paulapuro, H. (1996). "Quantification of water in different states of interaction with wood pulp fibers," Cellulose 3(4), 189-202.

Wolterink, J. K., Barkema, G. T., and Panja, D. (2006). "Passage times for unbiased polymer translocation through a narrow pore," Phys. Rev. Let. 96(20), article 208301.

Wu, N., Hubbe, M. A., Rojas, O. J., and Yamaguchi, T. (2008). "Penetration of highcharge cationic polymers into silica gel particles and cellulosic fibers," Proceedings of the $2^{\text {nd }}$ IPEC Conference, Tianjin, China, Book B, 626-649.

Yamamoto, N., Fujii, M., Kumagai, K., and Yanagisawa, Y. (2004). "Time course shift in particle penetration characteristics through capillary pore membrane filters," $J$. Aerosol Sci. 35(6), 731-741.

Zvikelsky, O., and Weisbrod, N. (2006). "Impact of particle size on colloid transport in discrete fractures," Water Resources Res. 42(12), article W12S08. 